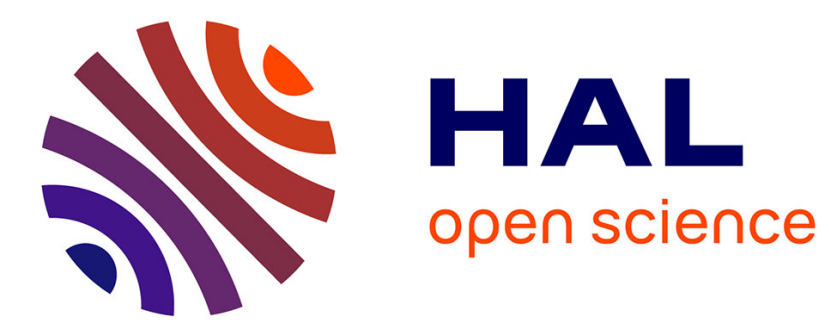

\title{
DISPERSIVE SCHEMES FOR THE CRITICAL KORTEWEG-DE VRIES EQUATION
}

Corentin Audiard

\section{To cite this version:}

Corentin Audiard. DISPERSIVE SCHEMES FOR THE CRITICAL KORTEWEG-DE VRIES EQUATION. Mathematical Models and Methods in Applied Sciences, 2013, Mathematical Models and Methods in Applied Sciences, 23 (14), pp.2603-2646. 10.1142/S0218202513500413 . hal-01157528

\section{HAL Id: hal-01157528 \\ https://hal.science/hal-01157528}

Submitted on 28 May 2015

HAL is a multi-disciplinary open access archive for the deposit and dissemination of scientific research documents, whether they are published or not. The documents may come from teaching and research institutions in France or abroad, or from public or private research centers.
L'archive ouverte pluridisciplinaire HAL, est destinée au dépôt et à la diffusion de documents scientifiques de niveau recherche, publiés ou non, émanant des établissements d'enseignement et de recherche français ou étrangers, des laboratoires publics ou privés. 


\title{
Dispersive schemes for the critical Korteweg de Vries equation
}

\author{
Corentin Audiard *†‡
}

\begin{abstract}
In this paper we study semi-discrete finite difference schemes for the critical Korteweg de Vries equation $(\mathrm{cKdV}$, which is gKdV for $k=4)$. We prove that the solutions of the discretized equation (using a two grid algorithm) satisfy dispersive estimates uniformly with respect to the discretization parameter. This implies convergence in a weak sense of the discrete solutions to the solution of the Cauchy problem even for rough initial data. We also prove a scattering result for the discrete equation, and show that the discrete scattering function converges to the continuous one. Finally rates of convergence are obtained for the approximation of a semi-linear equation with initial data in $H^{s}, s>0$, yet a similar result remains open for the quasi-linear ckdV equation. Our analysis relies essentially on the discrete Fourier transform and standard harmonic analysis on the real line.
\end{abstract}

\section{Introduction}

The well-posedness of the generalized Korteweg de Vries equation

$$
\left\{\begin{array}{l}
\partial_{t} u+\partial_{x}^{3} u+\partial_{x} u^{5} / 5=0 \\
\left.u\right|_{t=0}=u_{0}
\end{array}\right.
$$

for $L^{2}$ initial data (which corresponds to the critical scaling) was obtained by Kenig Ponce and Vega 12. Scattering for small initial data was also proved as a direct consequence of the existence theorem. This result was optimal in the sense that any other gKdV equation admits traveling wave solutions (that are a nonlinear phenomenon) with arbitrarily small $L^{2}$ norm. The existence of solutions in negative regularity Sobolev spaces was later obtained for the KdV equation [11, but our paper will only focus on the cKdV equation. The well-posedness relies on the existence of several sharp dispersive estimates. Namely if $V(t)$ is the group generated by the skew symmetric operator $-\partial_{x}^{3}$, Kenig Ponce and Vega proved

$$
\left\|\partial_{x} V(t) u_{0}\right\|_{L^{\infty}\left(\mathbb{R}_{x} ; L^{2}\left(\mathbb{R}_{t}\right)\right.} \lesssim\left\|u_{0}\right\|_{L^{2}\left(\mathbb{R}_{x}\right)},
$$

*BCAM - Basque Center for Applied Mathematics; Mazarredo, 14, E48009, Bilbao, Basque Country - Spain.

${ }^{\dagger}$ UPMC Univ Paris 06, UMR 7598, Laboratoire Jacques-Louis Lions, F-75005, Paris, France

${ }^{\ddagger}$ CNRS, UMR 7598, Laboratoire Jacques-Louis Lions, F-75005, Paris, France 


$$
\left\|V(t) u_{0}\right\|_{L^{5}\left(\mathbb{R}_{x} ; L^{10}\left(\mathbb{R}_{t}\right)\right)} \lesssim\left\|u_{0}\right\|_{L^{2}\left(\mathbb{R}_{x}\right)} .
$$

If one is not allowed to use dispersive estimates, semi-group technics only give well-posedness $H^{s}, s>3 / 2$, as was done by Kato [8] in 1979. It is thus essential that numerical schemes mimick correctly the fine properties of dispersive equations if one wishes to approximate solutions for rough initial data. Though travelling waves are problably the most well known solutions of gKdV equations and are very smooth, the approximation of very rough solutions can be important in some contexts. For example stochastic versions of the KdV equation appear in modellisation of plasma fluids, it has been studied theoretically [16] and numerically [2, 3] by Debussche and Printems. In this framework the initial data only belong to $L^{2}$.

As was pointed out by Ignat and Zuazua [7] in their work on the nonlinear Schrödinger equation, numerical schemes often fail to reproduce dispersive properties of solutions. The deep reason is that the symbol of the discretized differentiation operators do not behave in the same way as the symbol of the continuous one : in particular they display a lack of convexity (cancellation of the second order derivative of the symbol) and a lack of "slope" (cancellation of the first derivative), which are key ingredients for the proof of (D1|D2). To enlight the origin of such ingredients, let us outline the proof of the estimate (D1) as is done in [10]. By Fourier transform, we have

$$
\partial_{x} u(x)=\frac{1}{2 \pi} \int_{\mathbb{R}} e^{i x \xi} e^{i \xi^{3} t} \widehat{u_{0}}(\xi) d \xi=\frac{1}{2 \pi} \int_{\mathbb{R}} e^{i x \eta^{1 / 3}} e^{i \eta t} \widehat{u_{0}}\left(\eta^{1 / 3}\right) \frac{d \eta}{3 \eta^{1 / 3}},
$$

now using Parseval's identity in the time variable and reversing the change of variables

$$
\left\|\partial_{x} u(x, \cdot)\right\|_{L_{t}^{2}}^{2}=\frac{1}{18 \pi} \int_{\mathbb{R}}\left|\widehat{u_{0}}\right|^{2} \frac{d \eta}{\eta^{2 / 3}}=\frac{1}{18 \pi} \int_{\mathbb{R}}\left|\widehat{u_{0}}\right|^{2} 3 \xi^{2} \frac{d \xi}{\xi^{2}}=\frac{1}{3}\left\|u_{0}\right\|_{L^{2}}^{2} .
$$

Looking with attention at the calculi, it is clear that the only important point is that $\left(\xi^{3}\right)^{\prime} \asymp \xi^{2}$, or naively put the slope of the symbol is large for $\xi$ large. However the symbol of any discrete differentiation operator is periodic in $\xi$. In particular it cancels at several points ; this is a first obstruction to dispersion. Similarly it can be seen that the gain of integrability (D2) relies on the curvature (wether it is convexity or concavity does not matter) of the symbol, another feature not satisfied for discrete schemes.

At this point, it is worth mentionning that this kind of difficulty has been overcome by Nixon [14] in the case of fully implicit schemes. Indeed in this case the numerical dissipation is particularly strong at the frequencies where the symbol of the discretized operator fails to behave like the continuous one. Our aim here is to deal rather with non dissipative schemes discrete in space and continuous in time - for which dispersive estimates are a pure consequence of the space discretization.

Our approach follows the one developped by Ignat and Zuazua for the Schrödinger equation in [7, where they used a "two grid filtering" in order to eliminate the bad frequencies. The technics rely on basic harmonic and functional analysis, and we tried to keep a reasonnable balance between self-containedness and heaviness of the calculus : when proofs are slight 
modifications of already existing arguments we often point to a reference instead of repeating them, and if several quantities are bounded by the same method the precise argument is detailed only once.

Our main result is that there exists $N \in \mathbb{N}$ and an interpolation operator $\Pi: l^{2}(N h \mathbb{Z}) \rightarrow l^{2}(h \mathbb{Z})$ actually constructed for $N=6$ such that the semi-discrete finite difference problem

$$
\left\{\begin{array}{l}
\partial_{t} u_{n}+\partial_{h}^{3} u_{n}+\left(\partial_{h} \Pi E u\right)_{n}^{5} / 5=0,(n, t) \in \mathbb{Z} \times \mathbb{R}, \\
\left.u\right|_{t=0}=\Pi u_{0, h}
\end{array}\right.
$$

is globally well-posed for $u_{0, h} \in l^{2}(N h \mathbb{Z})$ sufficiently small, and the solution satisfies dispersive estimates analogous to (D1, D2) uniformly in $h$. The notations $E, \Pi, \partial_{h}$ and $\partial_{h}^{3}$ are defined in the first and second sections. The convergence of the discrete solution is proved by standard weak convergence/compactness arguments. Of course no rate of convergence in $h$ can be obtained if we work at the critical level of regularity $u_{0} \in L^{2}$, but we expect that rates of convergence in $h^{\alpha s}$ should be true for $u_{0} \in H^{s}$ for some $\alpha \leq 2 / 5$. Though we did not manage to obtain such results for the quasi-linear $\mathrm{cKdV}$ equation, it is proved for a simpler (semilinear) equation in the last section of the article.

Finally, let us remind that scattering for the continuous problem means that there exists a function $w \in L_{x}^{2}$ such that $\lim _{t \rightarrow+\infty}\|u(t)-V(t) w\|_{L^{2}} \rightarrow 0$, where $V(t)$ is the group generated by $-\partial_{x}^{3}$. In other words the solution of the quasilinear problem behaves asymptotically as a solution of the linear one. We prove here a discrete version of this result :

$$
\exists w_{h}: \lim _{t}\left\|u_{h}(t)-V_{h}(t) w_{h}\right\|_{L^{2}} \rightarrow 0, \text { where } V_{h}(t) \text { is generated by }-\partial_{h}^{3} .
$$

We prove also that $w_{h}$ converges to $w$ in a weak sense.

The paper is organized as follows:

- In section 1 we set up notations and basic notions used,

- Sections 2 and 3 are focused on the derivation of linear dispersive estimates for the semidiscrete problem. More precisely the discrete analog of local smoothing (D1) is obtained in section 2 while global gain of integrability (D2) is tackled in section 3.

- The well-posedness of the discrete problem in a convenient space is proved in section 4.

- We prove in section 5 that, provided the discretized initial data converge to $u_{0}$, then the solution of the discrete problem converges strongly in $L_{l o c}^{2}\left(\mathbb{R}_{x} \times \mathbb{R}_{t}\right)$ (and weakly in stronger spaces) to the solution of the Cauchy problem.

- The study of scattering is done in section 6 .

- Finally, section 7 deals with rates of convergence for a simpler (semilinear) problem when the initial data is in $H^{s}, s>0$. 
- The appendix describes discrete versions of well-known results of Fourier and harmonic analysis and contains a dispersive estimate that we did not manage to use for section 7 , but that may be useful in order to obtain rates of convergence for cKdV.

\section{Notations and basic properties}

The set of sequences defined on $h \mathbb{Z}$ is denoted $\mathcal{S}(h \mathbb{Z})$. As usual, the Lebesgue spaces on the real line are denoted $L^{p}(\mathbb{R})$ while the discrete Lebesgue spaces are $l^{p}(h \mathbb{Z})$, or in a shortened notation $l_{h}^{p}$, or $l^{p}$ when there is no ambiguity. The norm of a sequence $u \in l^{p}(h \mathbb{Z})$ is defined as

$$
\|u\|_{l^{p}}^{p}=h \sum\left|u_{j}\right|^{p} .
$$

We will index a sequence of $\mathcal{S}(h \mathbb{Z})$ as $u_{j h}$ or $u_{j}$ if there is no ambiguity. We write $\mathbb{R}_{t}, \mathbb{R}_{x}$ in order to avoid confusion between time and space, and write for conciseness $L_{x}^{p} L_{t}^{q}:=L^{p}\left(\mathbb{R}_{x} ; L^{q}\left(\mathbb{R}_{t}\right)\right)$, and similarly $l^{p} L^{q}, L_{t}^{p} L_{x}^{q}$. When working on bounded time intervals, and if there is no risk of confusion we write $L_{T}^{2}$ for $L^{2}([-T, T])$ or $L^{2}([0, T])$.

If $a, b$ are two numbers depending on several parameters the notation $a \asymp b$ means that there exists two constants $\alpha, \beta>0$ independant of the parameters such that

$$
\alpha a \leq b \leq \beta a .
$$

Similarly if $a, b \geq 0, a \lesssim b$ means that there exists $C>0$ independant of the parameters such that $a \leq C b$.

The Fourier transform is the application $u \mapsto \mathcal{F}(u)=\widehat{u}=\int_{\mathbb{R}} e^{-i x \xi} u(x) d x$, the discrete Fourier transform is the application

$$
\mathcal{S}(h \mathbb{Z}) \ni\left(u_{n}\right) \mapsto\left(\xi \mapsto \widehat{u}(\xi)=h \sum_{\mathbb{Z}} e^{-i j h \xi} u_{j}\right) .
$$

The sequence $\left(u_{j}\right)$ can be deduced from its Fourier transform thanks to the Fourier inversion formula

$$
u_{j}=\frac{1}{2 \pi} \int_{-\pi / h}^{\pi / h} e^{i j h \xi} \widehat{u}(\xi) d \xi:=\left(\mathcal{F}^{-1} \widehat{u}\right)_{j} .
$$

A Fourier multiplier $M(D)$ of symbol $m(\xi)$ is defined by $\widehat{M u}(\xi)=m(\xi) \widehat{u}(\xi)$. In particular, we may define the fractional derivative operators

$$
|D|^{s} u:=\mathcal{F}^{-1}\left(|\xi|^{s} \widehat{u}\right),
$$

and emphasize that this definition holds for sequences as well as for functions.

This leads to the definition of discrete and continuous Sobolev spaces for $s \geq 0$

$$
\begin{array}{r}
H^{s}(h \mathbb{Z})=\left\{\left(u_{n}\right) \in l^{2}(h \mathbb{Z}):\|u\|_{H^{s}}:=\left\|\left(1+|D|^{s}\right) u\right\|_{l^{2}}<\infty\right\}, \\
H^{s}(\mathbb{R})=\left\{u \in L^{2}(\mathbb{R}):\|u\|_{H^{s}}:=\left\|\left(1+|D|^{s}\right) u\right\|_{L^{2}}<\infty\right\} .
\end{array}
$$


We now turn to the symbol of discrete differentiation operators. The operator $\partial_{h}$ is defined by

$$
\left(\partial_{h} u\right)_{j h}=\frac{u_{(j+1) h}-u_{j h}}{h},
$$

it is a Fourier multiplier on $\mathcal{S}(h \mathbb{Z})$ with symbol $e^{i h \xi}-1$. In section 5 , we will also use $\partial_{h}$ as an operator on functions defined by $\left(\partial_{h} u\right)(x)=(u(x+h)-u(x)) / h$. We use in all the paper the standard discretization of the third order derivative operator

$$
\left(u_{j}\right) \rightarrow\left(\partial_{h}^{3} u_{h}\right)_{j}:=\frac{u_{h(j+2)}-2 u_{h(j+1)}+2 u_{h(j-1)}-u_{h(j-2)}}{2 h^{3}} .
$$

The symbol of $-\partial_{h}^{3}$ is

$$
i p_{h}(\xi)=i \frac{4}{h^{3}} \sin ^{2}(\xi h / 2) \sin (\xi h) .
$$

Note that (despite the notation) $\partial_{h}^{3} \neq \partial_{h} \circ \partial_{h} \circ \partial_{h}$. This is a stable (semi-)discretization for the Airy equation $\partial_{t}+\partial_{x}^{3} u=0$, indeed

$$
\partial_{t} \widehat{u}-i p_{h}(\xi) \widehat{u}(\xi) \Rightarrow \widehat{u}=e^{\left.i p_{h}(\xi) t\right)} \widehat{u_{0}},
$$

implying the conservation of the $l_{h}^{2}$ norm by the Parseval equality (and of any $H^{s}$ norm). The first order derivative of $p_{h}$ is

$$
p_{h}^{\prime}=4 \sin (\xi h / 2) \sin (3 \xi h / 2) / h^{2},
$$

its second order derivative is

$$
p_{h}^{\prime \prime}=2(2 \sin (2 \xi h)-\sin (\xi h)) / h .
$$

The group corresponding to $-\partial_{h}^{3}$ will be denoted $V_{h}(t): u \rightarrow \mathcal{F}^{-1}\left(e^{i t p_{h}} \widehat{u}\right)$, we also remind that in the introduction we denoted $V(t)$ the group corresponding to $-\partial_{x}^{3}$, namely the Fourier multiplier with symbol $e^{i t \xi^{3}}$.

\section{Discrete dispersive smoothing}

The lack of smoothing effect for the operator $V_{h}(t)$ and arbitrary initial data could be proved as was done in [7, 6] for the Schrödinger equation. This is due to the cancellation of the symbol $p_{h}^{\prime}=4 \sin (h \xi / 2) \sin (3 h \xi / 2) / h^{2}$ at $\pm 2 \pi / 3 h$.

The method that we will follow to tackle this issue consists in filtering the initial data and the nonlinearity in a way such that their spectrum is localized away from $\pm 2 \pi / 3 h$. Namely if $\left(u_{n}\right)$ is a sequence of $3 h \mathbb{Z}$, we define $\Pi u_{j}$ defined on $h \mathbb{Z}$ as follows

$$
\left\{\begin{array}{l}
\Pi u_{3 j}=u_{3 j}, \\
\Pi u_{3 j+1}=2 / 3 u_{3 j}+1 / 3 u_{3 j+3}, \\
\Pi u_{3 j+2}=1 / 3 u_{3 j}+2 / 3 u_{3 j+3} .
\end{array}\right.
$$


We also define $E_{3}$ as the canonical projector

$$
\begin{aligned}
E_{3}: \mathcal{S}(h \mathbb{Z}) & \mapsto \mathcal{S}(3 h \mathbb{Z}), \\
\left(u_{n}\right) & \mapsto\left(u_{3 n}\right) .
\end{aligned}
$$

A word of caution on notations: The operators $\Pi$ and $E_{3}$ as defined are of course linked to $h$, though it is clear that their definition poorly depends on it. In order to lighten the notations we have chosen not to display this dependance, although the symbols of operators does change according to their natural scaling (see the case of $\partial_{h}, \partial_{h}^{3}$ ).

We have

$$
\widehat{\Pi E_{3} u}=\frac{1}{9}(1+2 \cos (h \xi))^{2} \widehat{E_{3} u} .
$$

Indeed

$$
\begin{aligned}
\widehat{\Pi E_{3} u}=h \sum \Pi E_{3} u_{j} e^{-i j h \xi}= & 3 h / 3 \sum u_{3 j} e^{-3 i j h \xi}\left(1+2 / 3 e^{i h \xi}+2 / 3 e^{-i h \xi}+1 / 3 e^{2 i h \xi}\right. \\
& \left.+1 / 3 e^{-2 i h \xi}\right) \\
= & \frac{1}{3} \widehat{E_{3} u}(\xi)(1+4 / 3 \cos h \xi+2 / 3 \cos (2 h \xi)) \\
= & \frac{1}{9} \widehat{E_{3} u}(\xi)\left(1+4 \cos h \xi+4 \cos ^{2} h \xi\right) \\
= & \frac{1}{9} \widehat{E_{3} u}(\xi)(1+2 \cos h \xi)^{2} .
\end{aligned}
$$

Note that it is clear that $\left\|\Pi E_{3} u\right\|_{l^{p}(h \mathbb{Z})} \asymp\left\|E_{3} u\right\|_{l^{p}(3 h \mathbb{Z})} \leq 3^{1 / p}\|u\|_{l^{p}(h \mathbb{Z})}$, this fact will be used during the rest of the article without further notice.

\section{Proposition 1. (dispersive smoothing)}

For any $u \in l_{3 h}^{2}$,

$$
\sup _{j} \int_{\mathbb{R}}\left|\left(|D| V_{h}(t) \Pi u\right)_{j}\right|^{2} d t \lesssim\|u\|_{l_{3 h}^{2}}^{2}
$$

and the inhomogeneous counterpart stands for any $\left(g_{3 j}(t)\right)$

$$
\left\||D|^{2} \int_{0}^{t} V\left(t-t^{\prime}\right) \Pi g\left(t^{\prime}\right) d t^{\prime}\right\|_{l^{\infty} L^{2}} \lesssim\|g\|_{l_{3 h}^{1} L_{t}^{2}} .
$$

Proof. By scaling, it is sufficient to prove it for $h=1$. We have

$$
\begin{aligned}
V(t) \Pi u_{j} & =\int_{-\pi}^{\pi} e^{i x \xi} e^{i t p(\xi)} \frac{(1+2 \cos \xi)^{2}}{9}|\xi| \widehat{u} d \xi \\
& =\int_{-\pi}^{\pi} e^{i x \xi} e^{4 i t \sin ^{2}(\xi / 2) \sin \xi} \frac{(1+2 \cos \xi)^{2}}{9}|\xi| \widehat{\Pi u} d \xi
\end{aligned}
$$


On $]-2 \pi / 3,2 \pi / 3\left[,\left(\sin ^{2}(\xi / 2) \sin \xi\right)^{\prime}=\sin (\xi / 2) \sin (3 \xi / 2)>0\right.$, thus $p$ is a diffeormorphism and we may use the change of variables $\eta=4 \sin ^{2}(\xi / 2) \sin \xi$. To lighten the notations we write $\xi=\xi(\eta)$ and $d \xi=f(\eta) d \eta$. This gives

$$
\begin{aligned}
& \int_{-2 \pi / 3}^{2 \pi / 3} e^{i x \xi} e^{4 i t \sin ^{2}(\xi / 2) \sin \xi} \frac{(1+2 \cos \xi)^{2}}{9}|\xi| \widehat{u} d \xi=\int_{-3 \sqrt{3} / 8}^{3 \sqrt{3} / 8} e^{i x \xi(\eta)+i t \eta} \frac{(1+2 \cos \xi)^{2}}{9}|\xi| \widehat{u} \\
& f(\eta) d \eta \\
& =\int_{\mathbb{R}} 1_{[-3 \sqrt{3} / 8,3 \sqrt{3} / 8]} e^{i x \xi(\eta)+i t \eta} \frac{(1+2 \cos \xi)^{2}}{9}|\xi| \widehat{u} f(\eta) d \eta,
\end{aligned}
$$

which is an inverse Fourier transform in time. Plancherel's formula thus implies

$$
\left\|\int_{-3 \sqrt{3} / 8}^{3 \sqrt{3} / 8} e^{i x \xi(\eta)} e^{i t \eta} \frac{(1+2 \cos \xi)^{2}}{9}|\xi| \widehat{u} f(\eta) d \eta\right\|_{L_{t}^{2}}^{2}=\int_{-3 \sqrt{3} / 8}^{3 \sqrt{3} / 8} \frac{(1+2 \cos \xi)^{4}}{81}|\xi|^{2}|\widehat{u} f(\eta)|^{2} d \eta
$$

then reversing the change of variable $\eta=\eta(\xi)$ we get

$$
\int_{-3 \sqrt{3} / 8}^{3 \sqrt{3} / 8}(1+2 \cos \xi)^{4}|\xi|^{2}|\widehat{u} f(\eta)|^{2} d \eta=\int_{-2 \pi / 3}^{2 \pi / 3}(1+2 \cos \xi)^{4}|\xi|^{2}|\widehat{u}|^{2} \frac{d \xi}{|4 \sin (\xi / 2) \sin (3 \xi / 2)|} .
$$

The divisor $\sin (\xi / 2) \sin (3 \xi / 2)$ has a first order cancellation at $\pm 2 \pi / 3$ and a second order cancellation at 0 , but since it is easily seen that

$$
\frac{(1+2 \cos \xi)|\xi|^{2} \mid}{\sin (\xi / 2) \sin (3 \xi / 2)} \text { is uniformly bounded on }[-2 \pi / 3,2 \pi / 3] \text {, }
$$

this implies

$$
\begin{aligned}
\int_{-2 \pi / 3}^{2 \pi / 3}(1+2 \cos \xi)^{4}|\xi|^{2}|\widehat{u}|^{2} \frac{d \xi}{\sin (\xi / 2) \sin (3 \xi / 2)} & \lesssim \int_{-2 \pi / 3}^{2 \pi / 3}|1+2 \cos \xi|^{3}|\widehat{u}|^{2} d \xi \\
& \lesssim\|\widehat{u}\|_{L^{2}}^{2} \\
& \lesssim\|u\|_{l_{3 h}^{2}}^{2} .
\end{aligned}
$$

The same argument can be applied without further difficulties in the areas $[2 \pi / 3, \pi]$ and $[-\pi,-2 \pi / 3]$ where $p$ is also monotone, this gives (2.3).

The estimate (2.4) is slightly more technical and is based on the formula

$$
|D|^{2} \int_{0}^{t} V_{1}\left(t-t^{\prime}\right) \Pi g\left(t^{\prime}\right) d t^{\prime}=\int_{-\pi}^{\pi} \int_{\mathbb{R}} e^{i x \xi} \xi^{2} \frac{e^{i \tau t}-e^{i p(\xi) t}}{i(\tau-p(\xi))} \frac{(1+2 \cos (\xi))^{2}}{9} \check{g}(\xi, \tau) d \xi d \tau,
$$

where $\check{g}$ is the Fourier transform of $f$ with respect to both $x$ and $t$. For the detail of the argument we refer to [12, theorem 3.5. 
Remark 2. It is clear from the proof that the only thing needed for the boundedness of $\frac{(1+2 \cos \xi)^{4}|\xi|^{2}}{\sin (\xi / 2) \sin (3 \xi / 2)}$ is the first order cancellation of $(1+2 \cos \xi)^{4}$ at $\pm 2 \pi / 3 h$. Any other Fourier multiplier whose symbol cancels in such a way would have also worked. This fact is important since in the next section we will see that different interpolators must be used to gain integrability.

Corollary 1. The following "dual" estimates also hold:

$$
\begin{aligned}
\forall u \in l_{h}^{2}, \quad & \left\|\Pi^{*}|D| V(t) u\right\|_{l_{3 h}^{\infty} L_{t}^{2}} \lesssim\|u\|_{l^{2}}, \\
\forall g \in l_{3 h}^{1} L_{t}^{2}, \quad & \sup _{t}\left\||D| \int_{-\infty}^{\infty} V\left(t-t^{\prime}\right) \Pi g\left(t^{\prime}\right) d t^{\prime}\right\|_{l^{2}} \lesssim\|\Pi g\|_{l^{1} L^{2}}, \\
& \left\||D| \int_{0}^{t} V\left(t-t^{\prime}\right) \Pi g\left(t^{\prime}\right) d t^{\prime}\right\|_{l^{2}} \lesssim\|\Pi g\|_{l^{1} L^{2}([0, t])} .
\end{aligned}
$$

Proof. The inequality (2.7) is a direct duality consequence of (2.6), indeed

$$
\begin{aligned}
\left\||D| \int_{-\infty}^{\infty} V\left(t-t^{\prime}\right) \Pi g\left(t^{\prime}\right) d t^{\prime}\right\|_{l^{2}} & =\sup _{\|u\|_{l^{2}}=1} \sum_{j}|D| \int_{-\infty}^{\infty} V\left(t-t^{\prime}\right)(\Pi g)_{j}\left(t^{\prime}\right) d t^{\prime} u_{j} \\
& =\sup _{\|u\|_{l^{2}}=1} \sum_{j} \int_{-\infty}^{\infty} g_{3 j}\left(t^{\prime}\right)\left(\Pi^{*} V\left(t^{\prime}-t\right)|D| u\right)_{3 j} d t^{\prime} \\
& \leq\|g\|_{l^{1} L_{t}^{2}}\left\|\Pi^{*} V\left(t^{\prime}-t\right)|D| u\right\|_{l^{\infty} L_{t^{\prime}}} \\
& \leq\|g\|_{l^{1} L_{t}^{2}}\|u\|_{l^{2}}
\end{aligned}
$$

while (2.8) is deduced from (2.7) by replacing $g$ by $1_{[0, t]} g$.

It remains to prove (2.6). We remind that $\psi=(1+2 \cos (h \xi))^{2} / 9$ is the symbol of $\Pi$. From the duality formula

$$
\begin{aligned}
\forall(u, v) \in l^{2}(3 h \mathbb{Z}) \times l^{2}(h \mathbb{Z}), 2 \pi \sum_{j} \Pi u_{j} v_{j}= & \int_{-\pi / h}^{\pi / h} \psi \widehat{u} \widehat{v} \\
= & \int_{-\pi /(3 h)}^{\pi /(3 h)} \widehat{u}(\xi)(\psi \widehat{v}(\xi-2 \pi / 3)+\psi \widehat{v}(\xi) \\
& +\psi \widehat{v}(\xi+2 \pi / 3) d \xi
\end{aligned}
$$

we see that $2 \pi \widehat{\Pi^{*} v}=\psi \widehat{v}(\xi-2 \pi / 3)+\psi \widehat{v}(\xi)+\psi \widehat{v}(\xi+2 \pi / 3)$ so that

$$
\left(\Pi^{*}|D| V(t) u \widehat{)}=|\xi-2 \pi / 3| e^{i t p} \psi \widehat{u}(\xi-2 \pi / 3)+|\xi| e^{i t p} \psi \widehat{u}(\xi)+|\xi+2 \pi / 3| e^{i t p} \psi \widehat{u}(\xi+2 \pi / 3),\right.
$$

and the proof of Proposition 1 can be repeated identically because $\psi$ cancels at the appropriate points. 


\section{Global dispersive estimates}

A key estimate for solutions of the linear evolution equation $\partial_{t} u+\partial_{x}^{3} u=0$ is

$$
\|u\|_{L_{x}^{4} L_{t}^{\infty}} \lesssim\left\||D|^{1 / 4} u_{0}\right\|_{L^{2}}
$$

it relies on the fact that the second derivative of the symbol $\xi^{3}$ does not cancel on $\mathbb{R}$ outside 0 , which is obviously not the case for the discrete symbol $p_{h}(\xi)$ on $[-\pi / h, \pi / h]$. This estimate is sharp in the sense that it is scale invariant:

$$
\left\|u\left(\lambda x, \lambda^{3} t\right)\right\|_{L_{x}^{4} L_{t}^{\infty}}=\|u(x, t)\|_{L_{x}^{4} L^{\infty} t} / \lambda^{1 / 4},\left\||D|^{1 / 4} u_{0}(\lambda x)\right\|_{L_{x}^{2}}=\left\|u_{0}\right\|_{L^{2}} / \lambda^{1 / 4},
$$

and the same scale invariancy holds for sequences of $\mathcal{S}(\mathbb{Z})$ dilated in sequences of $\mathcal{S}(h \mathbb{Z})$. This will be used in order to reduce our proofs to the case $h=1$.

According to (1.6), the second derivative of the discrete symbol $p_{1}$ cancels at the points where

$$
2 \sin (2 \xi)=\sin \xi
$$

Except the obvious points $0, \pm \pi$, there are only two solutions $\left(\xi_{1}, \xi_{0}\right)$ in $]-\pi, \pi[$, and up to reindexing we can assume that $\left.\xi_{1} \in\right]-\pi / 2,-\pi / 4\left[\right.$ and $\left.\xi_{0} \in\right] \pi / 4, \pi / 2[$. Similarly to the previous section, we will see that the derivation of global dispersive estimates only requires to use an interpolation operator whose symbol cancels at $\left(\xi_{0}, \xi_{1}\right)$. Unfortunately they are not rational multiple of $\pi$, thus no 'barycentric' interpolator may be used to filter those frequencies as in the previous section.

We chose instead to use an interpolation operator more tailored to this case, namely an operator $\Pi$ such that

$$
\widehat{\Pi u}\left(\xi_{0}\right)=\widehat{\Pi u}\left(\xi_{1}\right)=\widehat{\Pi u}( \pm 2 \pi / 3)=\widehat{\Pi u}( \pm \pi)=0 .
$$

Given $u_{6 j}$ defined on the coarse grid $6 h \mathbb{Z}$, we set for $1 \leq k \leq 5$

$$
\Pi_{\alpha} u_{6 j+k}=\alpha_{k} u_{6 j}+\left(1-\alpha_{k}\right) u_{6(j+1)},
$$

the discrete Fourier transform of $\left(\Pi_{\alpha} u\right)_{j}$ is then

$$
\begin{aligned}
\widehat{\Pi_{\alpha} u}=\sum e^{-i j \xi} u_{j} & =\sum u_{6 j} e^{-6 i j \xi}+\sum_{k=1}^{5} e^{-i(6 j+k) \xi}\left(\alpha_{k} u_{6 j}+\left(1-\alpha_{k}\right) u_{6(j+1)}\right) \\
& =\sum e^{-i 6 j \xi} u_{6 j}\left(\sum_{k=0}^{5} e^{-i k \xi}+\alpha_{k} e^{-i k \xi}-\alpha_{k} e^{i(6-k) \xi}\right) \\
& =\frac{1}{6} \widehat{u}(\xi)\left(\frac{1-e^{i 6 \xi}}{1-e^{i \xi}}+\sum_{1}^{5} \alpha_{k} e^{-i k \xi}\left(1-e^{i 6 \xi}\right)\right) \\
& =\frac{1-e^{6 i \xi}}{6} \widehat{u}(\xi)\left(\frac{1}{1-e^{i \xi}}+\sum_{1}^{5} \alpha_{k} e^{-i k \xi}\right) \\
& =m(\xi) \widehat{u}(\xi) .
\end{aligned}
$$




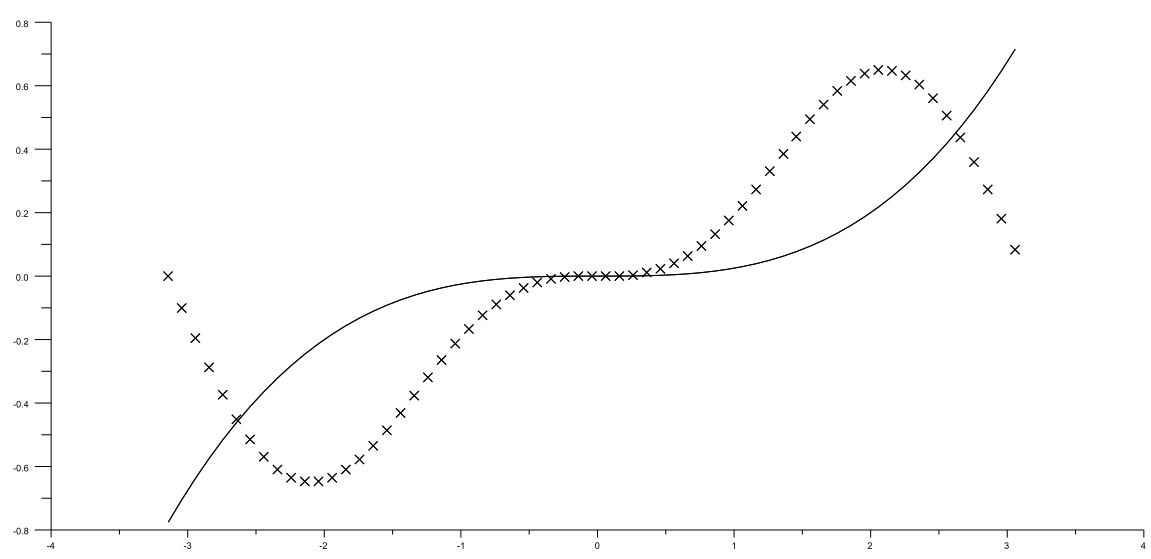

Figure 1: The connected line is the graph of the symbol of $-\partial_{x}^{3}$, the other one is the graph of the discrete symbol $p$ for $h=1$. The 'bad' points are the inflexion points $\xi_{0}, \xi_{1}$ and the local extremas $\pm 2 \pi / 3$.

It is clear that without restriction on $\alpha_{k}$ we have $m(0)=1, m(k \pi / 3)=0, k \neq \equiv 0(\bmod 6)$. The system

$$
\left\{\begin{array}{l}
1 \leq k \leq 5, m(2 k \pi / 6)=0, \\
m\left(\xi_{0}\right)=0 \\
m\left(\xi_{1}\right)=0
\end{array}\right.
$$

is thus underdetermined and we may arbitrarily choose a solution $\left(\alpha_{k}\right)$. The optimal choice of $\left(\alpha_{k}\right)$ (minimizing the norm of $\Pi_{\alpha}$ ) is a question that we will not study.

Remark 3. The construction was performed here for $h=1$, however it is clear that the same construction of an operator $\Pi_{\alpha}: \mathcal{S}(6 h \mathbb{Z}) \rightarrow \mathcal{S}(h \mathbb{Z})$ leads to an interpolator whose symbol is simply $m(h \xi)$, and thus cancels automatically at $k \pi / 3 h, k \neq \equiv 0(\bmod 6)$.

More generally, any interpolator $\mathcal{S}(N h \mathbb{Z}) \rightarrow \mathcal{S}(h \mathbb{Z})$ constructed by this technic has for symbol

$$
m_{h}(\xi)=\frac{1-e^{i N h \xi}}{N}\left(\frac{1}{1-e^{i h \xi}}+\sum_{1}^{N-1} \alpha_{k} e^{-i k h \xi}\right),
$$

and thus always cancels at $2 k \pi / N h, k \neq 0(\bmod N)$. This fact will be important for the sections dealing with convergence to the exact solution.

We simply assume in the rest of this section that an operator $\Pi: \mathcal{S}(N h \mathbb{Z}) \rightarrow \mathcal{S}(h \mathbb{Z})$ is given whose symbol has a first order cancellation at $\xi_{0}, \xi_{1}, \pm \pi$.

Proposition 4. Let $\Pi: N h \mathbb{Z} \rightarrow h \mathbb{Z}$ be a discrete Fourier multiplier, i.e. there exists a smooth function $\psi$ such that $\widehat{\Pi f}=\psi \widehat{f}$. If $\psi$ cancels at $\xi_{0} / h, \xi_{1} / h, \pm \pi / h$, then for any $u_{0} \in l^{2}(N h \mathbb{Z})$,

$$
\left\|V_{h}(t) \Pi u_{0}\right\|_{l_{j}^{4} L_{t}^{\infty}} \leq C\left\||D|^{1 / 4} u_{0}\right\|_{l^{2}}
$$


For any $f \in l_{N h}^{4 / 3} L_{t}^{1}$, we have the estimates

$$
\left\||D|^{-1 / 2} \int_{0}^{t} V_{h}(t-s) \Pi f(s) d s\right\|_{l^{4} L_{t}^{\infty}} \leq C\|f\|_{l^{4 / 3} L^{1}},
$$

In both estimates the constant $C$ does not depend on $h$.

Proof. The proof follows the one of the continuous case, up to some supplementary technical difficulties.

By duality and elementary calculus the inequality (3.4) can be reduced to

$$
\left\|\int_{\mathbb{R}}|D|^{-1 / 4} V_{h}(t) \Pi \Pi^{*} V\left(-t^{\prime}\right) D^{-1 / 4} g_{j}\left(t^{\prime}\right) d t^{\prime}\right\|_{l_{j}^{4} L_{t}^{\infty}} \lesssim\|g\|_{l_{j}^{4 / 3} L_{t}^{1}}
$$

(for a detailed argument see [13] Lemma 7.3). By repeating the argument of the proof of (2.6), we see that it is sufficient to prove the above estimate for the function $\int_{\mathbb{R}}|D|^{-1 / 2} V_{h}(t-$ $\left.t^{\prime}\right) \Pi \Pi^{*} g_{j}\left(t^{\prime}\right) d t^{\prime}$, but then we have

$$
\begin{aligned}
& \int_{\mathbb{R}}(\cdots) d t^{\prime}=\int_{-\pi / h}^{\pi / h} \int_{\mathbb{R}} \frac{e^{i\left(t-t^{\prime}\right) p_{h}(\xi)+i j \xi}}{|\xi|^{1 / 2}} \widehat{\Pi \Pi^{*} g}\left(\xi, t^{\prime}\right) d t^{\prime} d \xi \\
& =\int_{-\pi / h}^{\pi / h} \int_{\mathbb{R}} \psi \frac{e^{i\left(t-t^{\prime}\right) p_{h}(\xi)+i j \xi}}{|\xi|^{1 / 2}} \widehat{\Pi^{*} g}\left(\xi, t^{\prime}\right) d t^{\prime} d \xi \\
& \leq \int_{\mathbb{R}}\left|\left(\Pi^{*} g\right)_{j}\left(t^{\prime}\right)\right| d t^{\prime} *_{j} \sup _{t}\left|\int_{-\pi / h}^{\pi / h} \frac{e^{i t p_{h}(\xi)+i j \xi}}{|\xi|^{1 / 2}} \psi d \xi\right| \\
& \lesssim\left\|\max _{|j-k| \leq 5}\left|g_{k}\right|\right\|_{L_{t}^{1}} *_{j} \sup _{t}\left|\int_{-\pi / h}^{\pi / h} \frac{e^{i t p_{h}(\xi)+i j \xi}}{|\xi|^{1 / 2}} \psi d \xi\right|,
\end{aligned}
$$

the last inequality being a direct consequence of the explicit form of $\Pi^{*}$. Thus we are reduced to prove

$$
\|\| \max _{|j-k| \leq 5}\left|g_{k}\right|\left\|_{L^{1}(\mathbb{R})} *_{j} \sup _{t}\left|\int_{-\pi / h}^{\pi / h} \frac{e^{i t p_{h}(\xi)+i j \xi}}{|\xi|^{1 / 2}} \psi d \xi\right|\right\|_{l_{j}^{4}} \lesssim\|g\|_{l^{4 / 3} L^{1}}
$$

Similar arguments show that (3.5) amounts to

$$
\left\|\sup _{t}\right\| \max _{|j-k| \leq 5}\left|f_{k}\right|\left\|_{L^{1}(0, t)} *_{j} \sup _{s}\left|\int_{-\pi / h}^{\pi / h} \frac{e^{i s p_{h}(\xi)+i j \xi}}{|\xi|^{1 / 2}} \psi d \xi\right|\right\|_{l_{j}^{4}} \lesssim\|f\|_{l^{4 / 3} L^{1}},
$$

which is implied by (3.6). According to proposition 22 p 38 (discrete Hardy-LittlewoodSobolev), it is sufficient to prove

$$
\sup _{t}\left|\int_{-\pi / h}^{\pi / h} \frac{e^{i t p(h \xi) / h^{3}+i j h \xi}}{|\xi|^{1 / 2}} \psi(h \xi) d \xi\right| \lesssim \frac{1}{(h(1+|j|))^{1 / 2}} .
$$


By homogeneity we may reduce it to $h=1$, indeed the change of variable $\xi=\eta / h$ shows that (3.7) is equivalent to

$$
\frac{1}{\sqrt{h}} \sup _{t}\left|\int_{-\pi}^{\pi} \frac{e^{i t p(\eta) / h^{3}+i j \eta}}{|\eta|^{1 / 2}} \psi(\eta) d \eta\right| \lesssim \frac{1}{(h(1+|j|))^{1 / 2}}
$$

which amounts to

$$
\sup _{t}\left|\int_{-\pi}^{\pi} \frac{e^{i t p(\eta)+i j \eta}}{|\eta|^{1 / 2}} \psi(\eta) d \eta\right| \lesssim \frac{1}{1+|j|^{1 / 2}} .
$$

We remind that $p(\xi)=4 \sin ^{2}(\xi / 2) \sin \xi$, we perform the analysis only on $[0, \pi], t \geq 0$, the proof being the same on $[-\pi, 0]$ or $t \leq 0$. The estimate for $j=0$ is trivial, thus we assume $j \in \mathbb{Z}^{*}$. We split the interval $[0, \pi]$ in several parts:

$$
\begin{array}{r}
A_{1}=\left[0, t^{-1 / 3}\right], \\
A_{2}=A_{1}^{c} \cap\left\{\left|t p^{\prime}+j\right| \geq|j| / 2\right\}, \\
A_{3}=\left(A_{1} \cup A_{2}\right)^{c} .
\end{array}
$$

Note that if,$t^{-1 / 3} \geq \pi$ we only need to work on $A_{1}$, moreover in this case the integrand only runs over $[0, \pi]$, though we do not write it explicitly.

If $|j| \leq C_{0} t^{1 / 3}, C_{0}>0$ fixed, one has trivially $\left|\int_{A_{1}}(\cdots)\right| \lesssim 1 / \sqrt{j}$, else

$$
\int_{A_{1}} e^{i(t p+j \xi)} \psi / \sqrt{\xi} d \xi=\int_{0}^{1 /|j|} e^{i(t p+j \xi)} \psi / \sqrt{\xi} d \xi+\int_{1 /|j|}^{1 / t^{1 / 3}} e^{i(t p+j \xi)} \psi / \sqrt{\xi} d \xi
$$

the first integral is obviously bounded by $1 / \sqrt{|j|}$. After an integration by part we obtain for the second one

$$
\begin{aligned}
\left|\int_{1 /|j|}^{1 / t^{1 / 3}} e^{i(t p+j \xi)} \psi / \sqrt{\xi} d \xi\right| & \leq \frac{t^{1 / 6}}{\left|j+t p^{\prime}\left(t^{-1 / 3}\right)\right|}+\frac{\sqrt{|j|}}{j+t p^{\prime}(1 / j)} \\
& +\left|\int_{1 /|j|}^{1 / t^{1 / 3}} e^{i(j \xi+t p)} \frac{1}{\xi^{1 / 2}\left(j+t p^{\prime}\right)}\left(\frac{-\psi}{2 \xi}-\frac{\psi t p^{\prime \prime}}{\left(j+t p^{\prime}\right)}+\psi^{\prime}\right)\right| \\
& \lesssim \frac{1}{\sqrt{|j|}},
\end{aligned}
$$

provided $C_{0}$ is chosen large enough, for $\left|p^{\prime}\right| \lesssim \xi^{2}$ and $p^{\prime} \sim_{0} 3 \xi^{2}$. We implicitly used the fact that the integration interval is bounded to get the estimate

$$
\left|\int_{1 /|j|}^{1 / t^{1 / 3}} e^{i(j \xi+t p)} \frac{1}{\xi^{1 / 2}\left|j+t p^{\prime}\right|} \psi^{\prime}\right| \lesssim \int_{1 /|j|}^{1 / t^{1 / 3}} \frac{|j|^{1 / 2}}{\left|j+t p^{\prime}\right|} \lesssim \frac{1}{|j|^{1 / 2}}
$$


Let us note that the key point in the previous analysis was $j \geq C_{0} t^{1 / 3} \Rightarrow\left|j+t p^{\prime}\right| \gtrsim|j|$. Since on $A_{2}$ this inequality is automatically satisfied, the same integration by part argument gives

$$
\left|\int_{A_{2}} e^{i(t p+j \xi)} \psi / \sqrt{\xi} d \xi\right| \lesssim \frac{1}{\sqrt{|j|}}
$$

We still have to estimate the integral on $A_{3}$. Let us consider $\mathcal{V}, \widetilde{\mathcal{V}}$ two disjoint neighbourhoods of the points $\left\{\xi_{0}, \pi\right\}$ where $p^{\prime \prime}$ cancels, and set $A_{4}=A_{3} \cap(\mathcal{V} \cup \widetilde{\mathcal{V}})^{c}, A_{5}=A_{3} \cap \mathcal{V}, \widetilde{A_{5}}=A_{3} \cap \widetilde{\mathcal{V}}$. On $A_{4}, p^{\prime \prime} \asymp \xi, p^{\prime} \leq \xi^{2}$ and $t p^{\prime} \asymp j / 2$, thus $\xi \gtrsim \sqrt{|j| / t}$. The Van der Corput lemma (see [13] Corollary 1.1) implies

$$
\left|\int_{A_{4}} e^{i(t p+j \xi)} \psi / \sqrt{\xi} d \xi\right| \lesssim \frac{1}{\sqrt{t}} \sqrt[4]{\frac{t}{|j|}} \sup _{A_{4}} \frac{1}{\sqrt{\xi}} \lesssim \frac{1}{\sqrt{t}} \sqrt[4]{\frac{t}{|j|}}=\frac{1}{\sqrt{|j|}} .
$$

We then have on the neighbourhood of $\xi_{0}$

$$
p^{\prime}-p^{\prime}\left(\xi_{0}\right)=\frac{\beta}{2}\left(\xi-\xi_{0}\right)^{2}+o\left(\xi-\xi_{0}\right)^{2}, p^{\prime \prime}=\beta\left(\xi-\xi_{0}\right)+o\left(\xi-\xi_{0}\right), \beta \in \mathbb{R}^{*} .
$$

For $c$ (resp $C$ ) chosen small enough (resp large enough), if $t /|j| \notin[c, C]$, one obtains easily by integrations by parts

$$
\left|\int_{A_{5}} e^{i(t p+j \xi)} \psi / \sqrt{\xi} d \xi\right| \lesssim 1 /|j|
$$

thus we may assume that $t \asymp|j|$, and all that remains is to prove

$$
\left|\int_{A_{5}} e^{i(t p+j \xi)} \psi / \sqrt{\xi} d \xi\right| \lesssim 1 / \sqrt{t}
$$

This estimate can be seen as a particular case of lemma 2.7 in [9], we provide the argument for completeness. Since $\psi$ cancels at $\xi_{0}$, we have

$$
\left|\int_{\xi_{0}-1 / t^{1 / 3}}^{\xi_{0}+1 / t^{1 / 3}} e^{i(t p+j \xi)} \psi / \sqrt{\xi} d \xi\right| \lesssim t^{-2 / 3} \lesssim 1 / \sqrt{t},
$$

because $t$ is bounded away from 0 .

(note here that it would be enough that $\psi$ simply cancelled at the order $1 / 2$ )

On $A_{5}^{\prime}:=A_{5} \cap\left\{\left|p^{\prime}+j / t\right| \leq\left|\left(j+p^{\prime}\left(\xi_{0}\right) t\right) / 2 t\right|,\left|\xi-\xi_{0}\right| \geq t^{-1 / 3}\right\}$ one has $\left|\xi-\xi_{0}\right| \asymp \sqrt{\left(j+p^{\prime}\left(\xi_{0}\right) t\right) / 2 t}$ so that by using again the Van der Corput's lemma and the fact that $\psi \lesssim \max \left(\left|\xi-\xi_{0}\right|, 1\right) \Rightarrow$ $\psi \lesssim \sqrt{\left|\xi-\xi_{0}\right|}$ we get

$$
\begin{aligned}
\left|\int_{A_{5}^{\prime}} e^{i(t p+j \xi)} \psi / \sqrt{\xi} d \xi\right| \lesssim \frac{1}{\sqrt{t}}\left|\frac{j+p^{\prime}\left(\xi_{0}\right) t}{2 t}\right|^{-1 / 4} \sqrt{\frac{j+p^{\prime}\left(\xi_{0}\right) t}{2 t}} & =\frac{1}{\sqrt{t}}\left(\frac{j+p^{\prime}\left(\xi_{0}\right) t}{2 t}\right)^{1 / 4} \\
& \lesssim \frac{1}{\sqrt{t}} \lesssim \frac{1}{\sqrt{|j|}}
\end{aligned}
$$


Finally on $A_{5}^{\prime \prime}:=A_{5} \cap\left\{\left|p^{\prime}+j / t\right| \geq\left|\left(j+p^{\prime}\left(\xi_{0}\right) t\right) / 2 t\right|,\left|\xi-\xi_{0}\right| \geq t^{-1 / 3}\right\}$, one has $\left|p^{\prime}+j / t\right| \gtrsim$ $\left|p^{\prime}\right| \gtrsim\left|\xi-\xi_{0}\right|^{2} \geq t^{-2 / 3}$.

We may conclude again by integration by parts, since the calculus are very similar we shall only detail the case of one of the terms appearing :

$$
\left|\frac{1}{t} \int_{A_{5}^{\prime \prime}} \frac{e^{i(t p+x \xi)} \psi}{\left(p^{\prime}+j / t\right) \xi^{3 / 2}}\right| \lesssim \frac{1}{t} \int_{A_{5}^{\prime \prime}} \frac{\left|\xi-\xi_{0}\right|}{\left|\xi-\xi_{0}\right|^{2}} d \xi \lesssim \frac{1}{t} \max _{A_{5}^{\prime \prime}}\left|\ln \left(\left|\xi-\xi_{0}\right|\right)\right| \lesssim \frac{1}{\sqrt{|j|}}
$$

The integral estimate on $\widetilde{A_{5}}$ can be performed in the same way, and this concludes the proof.

Finally some other useful estimates are obtained by interpolation of the estimates in Prop. 4 and those of section 2. For the precise argument on interpolation we refer to [13] prop. 7.4.

Corollary 2. For $u_{0} \in l^{2}, f \in l^{5 / 4} L^{10 / 9}$

$$
\begin{array}{r}
\left\|V_{t}(t) \Pi u_{0}\right\|_{l^{5} L^{10}} \lesssim\left\|\Pi u_{0}\right\|_{l^{2}}, \\
\left\|\int_{0}^{t} V_{t}(t-s) \Pi f(s) d s\right\|_{l^{5} L^{10}} \lesssim\|f\|_{l^{5 / 4} L^{10 / 9}}, \\
\left\|D_{h} V_{t}(t) \Pi u_{0}\right\|_{l^{20} L^{5 / 2}} \lesssim\left\|D_{h}^{1 / 4} \Pi u_{0}\right\|_{l^{2}} .
\end{array}
$$

Remark 5. The results above apply to convenient interpolation operators, but also to any Fourier multiplier composed with any such interpolators. As a consequence we may (and will) also apply the estimates above to other operators, for instance $\partial_{h} \circ \Pi$.

\section{Existence of a solution}

This section is devoted to the existence of a global in time solution to the discrete dispersive scheme, that admits bounds depending only on the $l^{2}(h \mathbb{Z})$ of the initial data, and in particular independent of $h$. We first state a simple but useful lemma.

Lemma 1. In $H^{1}(h \mathbb{Z})$, the norm $\left(\int_{-\pi / h}^{\pi / h}|\widehat{u}|^{2}\left(1+|\xi|^{2}\right) d \xi\right)^{1 / 2}$ is (uniformly in $h$ ) equivalent to $\|u\|_{l^{2}}+\frac{\left\|u_{j+1}-u_{j}\right\|_{l^{2}}}{h}$.

The operator $\Pi \circ E_{k}$ is continuous $H^{s}(h \mathbb{Z}) \rightarrow H^{s}(h \mathbb{Z}), 0 \leq s \leq 1$, where $E_{k}$ is the natural projection operator $\mathcal{S}(h \mathbb{Z}) \rightarrow \mathcal{S}(k h \mathbb{Z}), \Pi$ is an interpolation operator as in the previous section.

Proof. A short computation shows that

$$
\widehat{u_{j+1}-} u_{j}=2 i e^{i h \xi / 2} \sin \frac{\xi h}{2} \widehat{u},
$$

and since on $[-\pi / h, \pi / h], 2 / h \sin \frac{\xi h}{2} \asymp \xi$, one has

$$
\|u\|_{H^{s}}^{2}=\int_{-\pi / h}^{\pi / h}|\widehat{u}|^{2}\left(1+|\xi|^{2}\right)^{s} d \xi \asymp \int|\widehat{u}|^{2}\left(1+\frac{2^{2 s} \sin ^{2 s} \frac{\xi h}{2}}{h^{2 s}}\right) d \xi,
$$


in particular for $s=1$

$$
\|u\|_{H^{1}} \asymp \int_{-\pi / h}^{\pi / h}|\widehat{u}|^{2}\left(1+4 \frac{\sin ^{2} \frac{\xi h}{2}}{h^{2}}\right) d \xi=\|u\|_{l^{2}}^{2}+\frac{\left\|u_{j+1}-u_{j}\right\|_{l^{2}}^{2}}{h^{2}} .
$$

It is easily checked that $\left\|\Pi \circ E_{k} u\right\|_{l^{2}} \lesssim\|u\|_{l^{2}}$. Moreover for $k n \leq j<k(n+1)$, by hypothesis $u_{j}=\alpha_{j} u_{k n}+\left(1-\alpha_{j}\right) u_{k(n+1)}$, so that

$$
\left|u_{j+1}-u_{j}\right| \leq\left|\alpha_{j+1}-\alpha_{j}\right| u_{k n}-u_{k(n+1)}\left|\leq\left(\max \left|\alpha_{j+1}-\alpha_{j}\right|\right) \sum_{1}^{5}\right| u_{k n+j}-u_{k n+j-1} \mid .
$$

This implies $\left\|\left(\Pi \circ E_{k} u\right)_{j+1}-\left(\Pi \circ E_{k} u\right)_{j}\right\|_{l^{2}} \lesssim\left\|u_{j+1}-u_{j}\right\|_{l^{2}}$, the operator $\Pi \circ E_{k}$ is thus continuous $l^{2} \rightarrow l^{2}$ et $H^{1} \rightarrow H^{1}$, so that by interpolation it is continuous $H^{s} \rightarrow H^{s}, 0 \leq s \leq 1$.

As an application of the estimates of the previous sections we shall prove the existence of a solution for the following semi-discrete problem

$$
\left\{\begin{array}{l}
\partial_{t} u+\partial_{h}^{3} u+\partial_{h} \Pi E u^{5} / 5=0 \\
\left.u\right|_{t=0}=\Pi u_{0}
\end{array}\right.
$$

We say that $u$ is a solution of (DcKdV) if it belongs to $C\left(\mathbb{R}_{t}, l^{2}\right) \cap l^{5} L^{1} 0\left(\mathbb{R}_{t}\right)$ and satisfies

$$
u(t)=V_{h}(t) \Pi u_{0}-\int_{0}^{t} V_{h}(t-s) \partial_{h} \Pi E u^{5} / 5 d s .
$$

Theorem 6. We define the space $X(\mathbb{R} \times h \mathbb{Z})$ as the set of functions $t \rightarrow u(t) \in \mathcal{S}(h \mathbb{Z})$ such that

$$
\begin{array}{r}
u \in \mathcal{C}\left(\mathbb{R} ; l^{2}\right) \cap L^{\infty}\left(\mathbb{R} ; l^{2}\right), \\
\left\|\partial_{h} u\right\|_{l^{\infty} L_{t}^{2}}<\infty, \\
\|u\|_{l^{5} L_{t}^{10}}<\infty .
\end{array}
$$

with the corresponding norm $\|u\|_{X}$ associated. We assume that $\Pi$ is an interpolation operator whose symbol cancels at the points $\pm 2 \pi / 3, \pm \pi, \xi_{0}, \xi_{1}$ (such operators exist according to the construction before Remark 0 (10).

There exists $\delta>0$ independent of $h$ such that for $\left\|\Pi u_{0}\right\|_{l^{2}}<\delta$ small enough there exists a unique solution $u$ of $(D c K d V$ satisfying

$$
\|u\|_{X}<\infty
$$

Moreover the solution map $\left\{u_{0} \in l^{2}(N h \mathbb{Z}):\left\|u_{0}\right\|_{l^{2}}<\delta\right\} \rightarrow X(h \mathbb{Z})$ is Lipschitz. 
Proof. The proof is based on the Picard-Banach fixed point theorem applied in the space $X$. We recall that $V_{h}(t)$ is the semigroup corresponding to the third order discretized derivative, it is a Fourier multiplier of symbol $e^{i t p_{h}(\xi)}$. We check that for $\left\|u_{0}\right\| \leq \delta$ and $a$ small enough,

$$
T: u \rightarrow V_{h}(t) \Pi u_{0}-\int_{0}^{t} V_{h}(t-s) \partial_{h} \Pi E u^{5}(s) / 5 d s,
$$

sends $\left\{u \in X:\|u\|_{X}<a\right\}=B_{X}(0, a)$ to itself.

Control of the $L^{\infty} l^{2}$ norm: We have $\left\|V_{h}(t) \Pi u_{0}\right\|_{l^{2}} \leq\left\|u_{0}\right\|_{l^{2}}$ and

$$
\left\|\int_{0}^{t} V_{h}(t-s) \partial_{h} \Pi E u^{5}(s) d s\right\|_{l^{2}} \lesssim\left\|\Pi E u^{5}\right\|_{l^{1} L^{2}} \lesssim\|u\|_{l^{5} L^{10}}^{5} \leq\|u\|_{X}^{5} .
$$

Control of $\left\|\partial_{h} T u\right\|_{l^{\infty} L^{2}}$ : Using Proposition 1 we may write

$$
\left\|\partial_{h} V_{h}(t) \Pi u_{0}\right\|_{l^{\infty} L^{2}} \lesssim\left\|u_{0}\right\|_{l^{2}},
$$

moreover

$$
\left\|\partial_{h}^{2} \int_{0}^{t} V_{h}(t-s) \Pi E u^{5}(s) d s\right\|_{l^{\infty} L^{2}} \lesssim\left\|\Pi E u^{5}\right\|_{L^{1} l^{2}} \lesssim\|u\|_{l^{5} L^{10}}^{5} \leq\|u\|_{X}^{5} .
$$

Control of the $l^{5} L^{10}$ norm: By Corollary 2, $\left\|V_{h}(t) \Pi u_{0}\right\|_{l^{5} L^{10}} \lesssim\left\|u_{0}\right\|_{l^{2}}$, and

$$
\left\|\int_{0}^{t} V_{h}(t-s) \partial_{h} \Pi E u^{5}(s) d s\right\|_{l^{5} L^{10}} \lesssim\left\|\partial_{h} \Pi E u^{5}\right\|_{l^{5 / 4} L^{10 / 9}} .
$$

Since

$$
(\Pi E u)_{j+1}^{5}-(\Pi E u)_{j}^{5}=\left((\Pi E u)_{j+1}-(\Pi E u)_{j}\right)\left(\sum_{0}^{4}(\Pi E u)_{j+1}^{k}(\Pi E u)_{j}^{4-k}\right),
$$

Hölder inequality implies

$$
\left\|\partial_{h} \Pi E u^{5}\right\|_{l^{5 / 4} L^{10 / 9}} \lesssim\left\|\partial_{h} \Pi E u\right\|_{l^{\infty} L^{2}}\left\|\Pi E u^{4}\right\|_{l^{5 / 4} L^{10 / 4}} .
$$

Using now the continuity of $\Pi \circ E: H^{1} \rightarrow H^{1}$ (lemma 1)

$$
\left\|\partial_{h} \Pi E u^{5}\right\|_{l^{5 / 4} L^{10 / 9}} \lesssim\left\|\partial_{h} u\right\|_{l^{\infty} L^{2}}\|u\|_{l^{5} L^{10}}^{4} \leq\|u\|_{X}^{5} .
$$

Finally we have obtained

$$
\|T u\|_{X} \leq c\left\|\Pi u_{0}\right\|_{l^{2}}+c\|u\|_{X}^{5} .
$$

Let $a$ be fixed such that $c a^{4}<1 / 2$, then if we choose $\delta \leq \frac{a}{2 c}$ we get

$$
\|T u\|_{X} \leq c\left\|\Pi u_{0}\right\|_{l^{2}}+a / 2 \leq a,
$$

that means $T: B_{X}(0, a) \rightarrow B_{X}(0, a)$. Following the same arguments, we see that (up to diminishing $a$ ) the operator is a contraction. By the Picard-Banach's fixed point theorem this ensures existence and uniqueness of a solution (in $X$ ) for any $u_{0}$ such that $\left\|u_{0}\right\|_{l^{2}} \leq \delta$. The smoothness of the solution operator follows from the classical fixed point theory. 


\section{Convergence to the solution of the Cauchy problem}

We denote by $P$ the interpolation operator from $l^{2}$ to the set of continuous affine by parts functions:

$$
\forall u \in l^{2}(h \mathbb{Z}), \forall x \in[j h,(j+1) h]: P u(x)=u_{j}+(x-j h) \frac{u_{j+1}-u_{j}}{h} .
$$

Although our natural level of regularity is only $l^{2}$, we need continuous functions in order to use the dispersive smoothing of $u_{h}$. The operator $P$ is particularly handy since it commutes with the operator $\partial_{h}$. Let $u_{h}$ be the discrete solution such that $P(\Pi u)_{0, h} \rightarrow u_{0}\left(L^{2}\right)$. The aim of this section is to establish the convergence of $P u_{h}$ to the solution of $(c K d V)$ in a sense which will be precised.

We start with a lemma that links the "smoothness" of a sequence $v$ to the smoothness of $P v$.

Lemma 2. For any $v \in \mathcal{S}(h \mathbb{Z})$, we have

$$
\|P v\|_{L^{2}(\mathbb{R})}=\|v\|_{l^{2}(h \mathbb{Z})},\|P v\|_{H^{1}(\mathbb{R})}=\left(\|v\|_{l^{2}}^{2}+\left\|\frac{v_{j+1}-v_{j}}{h}\right\|_{l^{2}}^{2}\right)^{1 / 2},
$$

and the same equalities are true if we replace $\mathbb{R}$ by $[k h, l h]$ and $h \mathbb{Z}$ by $k h \cdots l h$ for $(k, l) \in \mathbb{Z}^{2}$. The Fourier transform of $P u$ is

$$
\widehat{P v}(\xi)=\frac{4 \sin ^{2}(h \xi / 2)}{h^{2} \xi^{2}} \widehat{v}(\xi) .
$$

If $P \Pi v_{h} \rightarrow v\left(L^{2}\right)$, then for any fixed $t P V_{h}(t) \Pi v_{h} \rightarrow V(t) v\left(L^{2}\right)$, where $V$ is the semigroup corresponding to the operator $\partial_{x}^{3}$. Moreover if $v$ is fixed and $t$ remains in a compact the convergence is uniform.

Proof. The norm equalities are elementary. A simple calculation gives for the Fourier transform

$$
\begin{aligned}
\widehat{P v}(\xi) & =\sum_{j} \int_{j h}^{(j+1) h} e^{-i x \xi}\left(v_{j}+\frac{v_{j+1}-v_{j}}{h}(x-j h)\right) d x \\
& =\sum_{j} v_{j} e^{-i j h \xi} \frac{e^{-i h \xi}-1}{-i \xi}+\frac{v_{j+1}-v_{j}}{h}\left(\frac{h e^{-i j \xi}}{-i \xi}+\frac{e^{-i j h \xi}\left(e^{-i h \xi}-1\right)}{\xi^{2}}\right) \\
& =\sum_{j} \frac{v_{j+1}-v_{j}}{h} \frac{e^{-i j h \xi}\left(e^{-i h \xi}-1\right)}{\xi^{2}} \\
& =\frac{\left(e^{i h \xi}-1\right)\left(e^{-i h \xi}-1\right)}{h^{2} \xi^{2}} \widehat{v}(\xi)=\frac{4 \sin ^{2}(h \xi / 2)}{h^{2} \xi^{2}} \widehat{v}(\xi) .
\end{aligned}
$$


For the norm convergence we may write

$$
\begin{aligned}
& P \widehat{V_{h}(t) \Pi} v_{h}=\frac{4 \sin ^{2}(h \xi / 2)}{h^{2} \xi^{2}} e^{i t p_{h}(\xi)} \widehat{\Pi v_{h}}(\xi) \\
& \Rightarrow\left\|P V_{h}(t) \Pi v_{h}-V(t) v\right\|_{L^{2}}=\left(\int_{\mathbb{R}}\left|\frac{4 \sin ^{2}(h \xi / 2)}{h^{2} \xi^{2}} e^{i t p_{h}} \widehat{\Pi v_{h}}(\xi)-e^{i t \xi^{3}} \widehat{v}\right|^{2} d \xi\right)^{1 / 2} \\
& \leq\left(\int_{\mathbb{R}}\left|\frac{4 \sin ^{2}(h \xi / 2)}{h^{2} \xi^{2}} e^{i t p_{h}} \widehat{\Pi v_{h}}(\xi)-e^{i t p_{h}} \widehat{v}\right|^{2} d \xi\right)^{1 / 2} \\
& +\left(\int_{\mathbb{R}}\left|\left(e^{i t p_{h}}-e^{i t \xi^{3}}\right) \widehat{v}(\xi)\right|^{2} d \xi\right)^{1 / 2} .
\end{aligned}
$$

Since $\left|e^{i t p_{h}}\right|=1$, the first term tends to 0 according the the hypothesis $P \Pi v_{h} \rightarrow v\left(L^{2}\right)$, moreover $\left|e^{t p_{h}}-e^{i t \xi^{3}}\right| \leq 2$ and $\rightarrow_{h \rightarrow 0} 0$, so that by Lebesgue's dominated convergence theorem the second term also tends to 0 .

If $t, \xi$ remain in a compact, $\left|t\left(p_{h}(\xi)-\xi^{3}\right)\right| \rightarrow 0$ uniformly. Since $\lim _{A \rightarrow \infty}\|\widehat{v}\|_{L^{2}(|\xi| \geq A)}=0$, the uniform convergence of $P V_{h}(t) \Pi v_{h}$ to $V(t) v$ follows easily.

Remark 7. The convergence $P V_{h}(t) \Pi u_{h} \rightarrow V(t) u\left(L^{2}\right)$ is uniform in $t$ for $t$ bounded, however it does depend on $u_{0}$, preventing any rate of convergence in $h$.

In what follows we shall assume that $u_{0 h} \in l^{2}(N h \mathbb{Z})$ is such that

$$
\left\|P \Pi u_{0 h}-u_{0}\right\|_{L^{2}} \rightarrow_{h} 0,
$$

which is clearly the minimal assumption for convergence, and we consider the family $u_{h}$ of solutions to

$$
\left\{\begin{array}{l}
\partial_{t} u_{h}+\partial_{h}^{3} u_{h}+\partial_{h} \Pi E u_{h}^{5} / 5=0 \\
\left.u_{h}\right|_{t=0}=\Pi u_{0 h}
\end{array}\right.
$$

According to Theorem [6, the family $\left(P u_{h}\right)_{0 \leq h \leq 1}$ is bounded in $L_{t}^{\infty} L_{x}^{2} \cap L_{x}^{5} L_{t}^{10}$, and $\left(u_{h}(\cdot+\right.$ $\left.h)-u_{h}(\cdot)\right) / h$ is bounded in $L_{x}^{\infty} L_{t}^{2}$. By weak star (resp weak) compactness, we may extract $u_{h}$ weakly converging toward some $u \in L_{t}^{\infty} L_{x}^{2} \cap L_{x}^{5} L_{t}^{10}$.

It is slightly more delicate to check that $\partial_{x} u \in L_{x}^{\infty} L_{t}^{2}$. We use that

$$
\forall \varphi \in C_{c}^{\infty}, \int_{\mathbb{R}^{2}} \frac{P u_{h}(x+h)-P u_{h}(x)}{h} \varphi d x d t=\int_{\mathbb{R}^{2}} P u_{h}(x) \frac{\varphi(x-h)-\varphi(x)}{h} d x d t .
$$

Since $(\varphi(x-h)-\varphi(x)) / h \rightarrow-\varphi^{\prime} L_{x}^{1} L_{t}^{2}$, we have

$$
\int_{\mathbb{R}^{2}} P u_{h}(x) \frac{\varphi(x-h)-\varphi(x)}{h} d x d t \rightarrow-\int_{\mathbb{R}} u \varphi^{\prime} d x d t,
$$

Moreover up to extracting again we may assume that $\left(P u_{h}(\cdot+h)-P u_{h}(\cdot)\right) / h \rightarrow^{*} v \in L_{x}^{\infty} L_{t}^{2}$, so that

$$
\int_{\mathbb{R}^{2}} v \varphi d x d t=-\int_{\mathbb{R}^{2}} u \partial_{x} \varphi d x d t
$$


that is $\partial_{x} u=v \in L_{x}^{\infty} L_{t}^{2}$ (in the sense of distributions).

Theorem 8. Let $\Pi u_{0, h}$ be the initial data of the discrete problem (DcKdV). Assume that $P \Pi u_{0, h} \rightarrow_{h} u_{0}$ in $L^{2}$. Let $u \in L_{t}^{\infty} L_{x}^{2} \cap L_{x}^{5} L_{t}^{10}$ be the weak limit of any extracted subsequence of $P u_{h}$. Then $u$ is the solution of $c K d V$ with initial data $u_{0}$ and the whole sequence P $u_{h}$ converges to $u$ in the following sense

$$
\begin{array}{r}
P u_{h} \rightarrow^{*} u\left(L_{t}^{\infty} L_{x}^{2}\right), P u_{h} \rightarrow u\left(L_{x}^{5} L_{t}^{10}\right), \\
\partial_{x} P u_{h} \rightarrow^{*} \partial_{x} u\left(L_{x}^{\infty} L_{t}^{2}\right), P u_{h} \rightarrow u\left(L_{l o c}^{2}\left(\mathbb{R}^{2}\right)\right) .
\end{array}
$$

Proof. Let $T>0$ be fixed, $J=[-T, T], \Omega=[-C, C]$. Up to increasing by at most $2 h$ the size of $\Omega$ we may always assume that $C$ is a multiple of $h$ and apply the norm equalities of Lemma 2 (a fact that we will not mention in the rest of the proof). Since

$$
\partial_{t} P u_{h}=-P \partial_{h}^{3} u_{h}-P \partial_{h} \Pi E u_{h}^{5} / 5,
$$

we have according to the injection $L^{1}(\Omega) \hookrightarrow H^{-1}(\Omega)$ (dual of $H^{1} \hookrightarrow L^{\infty}$ )

$$
\begin{aligned}
\left\|\partial_{t} P u_{h}\right\|_{L^{1}\left(J, H^{-3}(\Omega)\right)} & \leq\left\|P u_{h}\right\|_{L^{1}\left(J, L^{2}\right)}+\left\|P \partial_{h} \Pi E u_{h}^{5} / 5\right\|_{L^{1}\left(J, H^{-3}(\Omega)\right)} \\
& \lesssim\left\|u_{0}\right\|_{L^{2}}+\left\|P \partial_{h} \Pi E u_{h}^{5} / 5\right\|_{L^{1}(J \times \Omega)} .
\end{aligned}
$$

As $J$ and $\Omega$ are bounded, we may write

$$
\left\|P \partial_{h} \Pi E u_{h}^{5}\right\|_{L^{1}(J \times \Omega)} \lesssim\left\|P \partial_{h} \Pi E u_{h}^{5}\right\|_{L_{x}^{5 / 4}\left(\mathbb{R}_{x}, L_{t}^{10 / 9}(J)\right)},
$$

then by application of Hölder's inequality and continuity of $\Pi E$ and $P$ :

$$
\left\|P \partial_{h} \Pi E u_{h}^{5}\right\|_{L_{x}^{5 / 4}\left(\mathbb{R}_{x}, L_{t}^{10 / 9}(J)\right)} \lesssim\left\|\partial_{h} u_{h}\right\|_{l^{\infty} L^{2}}\left\|u_{h}\right\|_{l^{5} L^{10}}^{4} \lesssim\left\|u_{0}\right\|_{L^{2}}^{5} .
$$

Thus $\partial_{t} P u_{h}$ is bounded in $L^{2}\left(J, H^{-3}(\Omega)\right)$. On the other hand

$$
\left\|P u_{h}\right\|_{L^{2}\left(J ; H^{1}(\Omega)\right)} \lesssim\left\|P u_{h}\right\|_{L^{\infty}\left(J ; L^{2}\right)}+\left\|\partial_{x} P u_{h}\right\|_{L_{x}^{\infty} L_{t}^{2}}
$$

and thus $P u_{h}$ is bounded in $L^{2}\left(J, H^{1}(\Omega)\right)$. Since we have the sequence of injections $H^{1}(\Omega) \hookrightarrow$ $L^{2}(\Omega) \hookrightarrow H^{-3}(\Omega)$ where the first injection is compact, Aubin-Lions's lemma implies that $P u_{h}$ is precompact in $L^{2}\left(J, L^{2}(\Omega)\right)$. Using a diagonal extraction argument, we find that a subsequence of $u_{h}$ is strongly converging to $u$ in $L_{\text {loc }}^{2}\left(\mathbb{R}_{x} \times \mathbb{R}_{t}\right)$ as $h \rightarrow 0$.

This list of convergences is now sufficient to check that the limit is the solution of the continuous problem. In order to do so we introduce the 'variational' formulation

$$
\forall \varphi \in C_{c}^{\infty}\left(\mathbb{R}^{2}\right), \int_{\mathbb{R}^{2}} P u_{h}\left(-\partial_{t} \varphi-\partial_{h}^{3} \varphi\right)-P \Pi E u_{h}^{5} \partial_{h} \varphi d x d t=0 .
$$


Passing to the limit in $h$ we will obtain that $u$ is a solution of $(\mathrm{cKdV})$. The linear terms are easily handled, indeed for $\varphi \in C_{c}^{\infty}, \partial_{h}^{3} \varphi \rightarrow \partial_{x}^{3} \varphi\left(L^{2}\right)$, thus using $P u_{h} \rightarrow u\left(L_{l o c}^{2}\right)$ we find

$$
\int_{\mathbb{R}^{2}} P u_{h}\left(-\partial_{t} \varphi-\partial_{h}^{3} \varphi\right) \rightarrow \int_{\mathbb{R}^{2}} u\left(-\partial_{t} \varphi-\partial_{x}^{3} \varphi\right) .
$$

For the nonlinear term, let us fix $R$ such that $\operatorname{supp} \varphi \subset]-R, R\left[^{2}\right.$ and $\varepsilon>0$ to be small. Let us define

$$
A_{h, \varepsilon}:=\left\{(x, t) \in[-R, R]^{2}:\left|u_{h}^{5}-u^{5}\right|>\varepsilon\right\} .
$$

Since $P u_{h} \rightarrow u L^{2}\left([-R, R]^{2}\right)$, up to an other extraction $P u_{h} \rightarrow u$ a.e. and we have for $\varepsilon>0$ fixed $\lambda\left(A_{h, \varepsilon}\right) \rightarrow_{h} 0$. For $h$ small enough we may write

$$
\int_{[-R, R]^{2}} \partial_{h} P \Pi E u_{h}^{5} \varphi d x d t=\int_{[-R, R]^{2}} P \Pi E u_{h}^{5} \partial_{-h} \varphi d x d t .
$$

We have $\partial_{-h} \varphi \rightarrow-\partial_{x} \varphi\left(L^{\infty}\left(\mathbb{R}^{2}\right)\right)$ thus is is sufficient to prove

$$
\int_{[-R, R]^{2}}\left(P \Pi E u_{h}^{5}-u^{5}\right) \partial_{-h} \varphi d x d t \rightarrow 0 .
$$

Let $N \in \mathbb{N}^{*}$ be such that $\Pi: N h \mathbb{Z} \rightarrow h \mathbb{Z}, \mathbb{Z}=\sqcup_{p=0}^{N-1}\{N h \mathbb{Z}+p h\}=: \sqcup \mathbb{Z}_{p}$ :

$$
\begin{aligned}
\left|\int_{[-R, R]^{2}}\left(P \Pi E u_{h}^{5}-u^{5}\right) \partial_{-h} \varphi d x d t\right| & \lesssim \int_{-R}^{R} \sum_{k \in \mathbb{Z}} \sum_{p=0}^{N-1} \int_{N k h+p}^{N k h+p+1}\left|\Pi E u_{N k h+p h}^{5}-u^{5}\right|\left|\partial_{-h} \varphi\right| d x d t \\
& \leq \int_{-R}^{R} \sum_{p, k} \int_{N k h+p}^{N k h+p+1} \sum_{j=0}^{1} \alpha_{j, p}\left|u_{N(k+j) h}^{5}-u^{5}\right|\left|\partial_{-h} \varphi\right| d x d t,
\end{aligned}
$$

where the $\alpha_{j, p}$ only depend on the operator $\Pi$. This implies

$$
\begin{aligned}
\left|\int_{[-R, R]^{2}}\left(P \Pi u_{h}^{5}-u^{5}\right) \partial_{-h} \varphi d x d t\right| & \leq \sum_{p=0}^{N-1} \int_{-R}^{R} \sum_{\mathbb{Z}_{p}} \sum_{j=0}^{1} \alpha_{j, p}\left|u_{N(k+j) h}^{5}-u^{5}\right|\left|\partial_{-h} \varphi\right| d x d t \\
\leq & \sum_{p=0}^{N-1} \sum_{j=0}^{1} \int_{-R}^{R} \int_{\mathbb{R}} \alpha_{j, p}\left|P u_{h}^{5}(x)-u^{5}\left(x+\tau_{j, p} h\right)\right|\left|\partial_{-h} \varphi\right| d x d t
\end{aligned}
$$

where $\tau_{j, p}=p$ if $j=0, p-N$ if $j=1$. Since $u \in L^{5} L^{10}$,

$$
\limsup _{\varepsilon \rightarrow 0} \int_{[-R, R]^{2}}\left|u^{5}(x+\varepsilon)-u^{5}(x)\right| d x d t=0,
$$

and we are reduced to prove

$$
\int_{-R}^{R} \int_{\mathbb{R}}\left|P u_{h}^{5}(x)-u^{5}(x)\right|\left|\partial_{-h} \varphi\right| d x d t=\int_{[-R, R]^{2}}\left|P u_{h}^{5}(x)-u^{5}(x)\right|\left|\partial_{-h} \varphi\right| d x d t \rightarrow 0 .
$$


But

$$
\begin{aligned}
\int_{[-R, R]^{2}}\left|P u_{h}^{5}(x)-u^{5}(x)\right|\left|\partial_{-h} \varphi\right| d x d t= & \int_{A_{h, \varepsilon}}\left|P u_{h}^{5}(x)-u^{5}(x)\right|\left|\partial_{-h} \varphi\right| d x d t . \\
& +\int_{A_{h, \varepsilon}^{c}}\left|P u_{h}^{5}(x)-u^{5}(x)\right|\left|\partial_{-h} \varphi\right| d x d t
\end{aligned}
$$

The second term is simply bounded by

$$
\left|\int_{A_{h, \varepsilon}^{c}}\right| P u_{h}^{5}(x)-u^{5}(x)|| \partial_{-h} \varphi|d x d t| \leq \varepsilon 4 R^{2} \sup _{0 \leq h \leq 1,(x, t) \in[-R, R]^{2}}\left|\partial_{h} \varphi\right| .
$$

For the other term we use a discrete version of the Sobolev embedding $H^{1} \hookrightarrow L^{\infty}$ (the proof is similar)

$$
P u_{h}, P \partial_{h} u_{h} \in L^{2}\left([-R, R]^{2}\right) \Rightarrow P u_{h} \in L_{t}^{2} L_{x}^{\infty}\left([-R, R]^{2}\right) \Rightarrow P u_{h} \in L_{x}^{\infty} L_{t}^{2}\left([-R, R]^{2}\right),
$$

with bound independent of $h$. By interpolating with $P u_{h} \in L_{x}^{5} L_{t}^{10}$ we get $P u_{h} \in L^{6}\left([-R, R]^{2}\right)$. The same (simpler) argument implies $u \in L^{6}\left([-R, R]^{2}\right)$, so that

$$
\int_{A_{h, \varepsilon}}\left|P \Pi u_{h}^{5}-u^{5}\right|\left|\partial_{-h} \varphi\right| d x d t \leq\left(\left\|P u_{h}^{5}\right\|_{L^{6 / 5}\left(A_{h, \varepsilon}\right)}^{5 / 6}+\left\|u^{5}\right\|_{L^{6 / 5}\left(A_{h, \varepsilon}\right)}^{5 / 6}\right)\left\|\partial_{-h} \varphi\right\|_{L^{6}\left(A_{h, \varepsilon}\right)}^{1 / 6},
$$

but since $\lambda\left(A_{h, \varepsilon}\right) \rightarrow 0$, we get

$$
\int_{A_{h, \varepsilon}}\left|P u_{h}^{5}-u^{5}\right|\left|\partial_{-h} \varphi\right| d x d t \rightarrow 0
$$

Finally we have obtained

$$
\forall \varepsilon>0, \limsup _{h \rightarrow 0}\left|\int_{[-R, R]^{2}}\left(P \Pi u_{h}^{5}-u^{5}\right) \partial_{h} \varphi d x d t\right| \leq 4 R^{2} \varepsilon \sup _{0 \leq h \leq 1,(x, t) \in[-R, R]^{2}}\left|\partial_{h} \varphi\right|,
$$

which means as desired $\partial_{h} P \Pi u_{h}^{5} \rightarrow \partial_{x} u^{5}\left(\mathcal{D}^{\prime}\right)$.

We have proved that and $u$ is a solution of $(c K d V)$. It remains to check is that this solution has indeed $u_{0}$ for initial data, that is $u \in C_{t} L^{2},\left.u\right|_{t=0}=u_{0}$. According to lemma 2 :

$$
\lim _{h, t \rightarrow 0} P V_{h}(t) \Pi u_{0, h}=u_{0}\left(L^{2}\right)(\text { see lemma 21), }
$$

and we must prove $\lim _{h, t \rightarrow 0}\left\|\int_{0}^{t} \partial_{h} V_{h}(t-s) \Pi u_{h}^{5}(s) d s\right\|_{L^{2}}=0$. According to the estimates of section 4 , we have

$$
\left\|\int_{0}^{t} \partial_{h} V_{h}(t-s) \Pi E u_{h}^{5}(s) d s\right\|_{L^{2}} \leq\left\|u_{h}^{5}\right\|_{l^{1} L^{2}([0, t])}=\left\|u_{h}\right\|_{l^{5} L^{10}([0, t])}^{5} .
$$


Moreover (following the proof of Theorem 6)

$$
\begin{aligned}
\left\|u_{h}\right\|_{l^{5} L^{10}([0, t])} & \leq\left\|V_{h} u_{0, h}\right\|_{l^{5} L^{10}([0, t])}+\left\|\int_{0}^{r} \partial_{h} V_{h}(r-s) \Pi E u_{h}^{5}(s) / 5 d s\right\|_{l^{5} L^{10}([0, t])} \\
& \leq\left\|V_{h} u_{0, h}\right\|_{l^{5} L^{10}([0, t])}+\left\|\partial_{h} \Pi E u_{h}^{5} / 5\right\|_{l^{5 / 4} L^{10 / 9}([0, t])} \\
& \leq\left\|V_{h} u_{0, h}\right\|_{l^{5} L^{10}([0, t])}+C\left\|\partial_{h} u_{h}\right\|_{l^{\infty} L^{2}}\left\|u_{h}\right\|_{l^{5} L^{10}([0, t])}^{4} \\
& \leq\left\|V_{h} u_{0, h}\right\|_{l^{5} L^{10}([0, t])}+\frac{1}{2}\left\|u_{h}\right\|_{l^{5} L^{10}([0, t])},
\end{aligned}
$$

so that $\left\|u_{h}\right\|_{l^{5} L^{10}([0, t])} \leq 2\left\|V_{h}(s) u_{0, h}\right\|_{l^{5} L^{10}([0, t])}$ and it is sufficient to prove that this last quantity goes to 0 when $h, t \rightarrow 0$. Let $\varepsilon>0$ be fixed, we introduce $v_{0} \in H^{1}$ such that $\left\|u_{0}-v_{0}\right\|_{L^{2}} \leq \varepsilon$, and we set $v_{0, h, n}=v_{0}(n h)$. Classically $\left\|P v_{0, h}-v_{0}\right\|_{H^{1}} \rightarrow 0$, this implies both $\left\|v_{0, h}-u_{0, h}\right\|_{l^{2}} \rightarrow 0$ and $\left\|P v_{0, h}\right\|_{H^{1}} \leq C\left\|v_{0}\right\|_{H^{1}}$. We get then, using the continuity of $V_{h}: H^{1}(h \mathbb{Z}) \rightarrow H^{1}(h \mathbb{Z})$,

$$
\begin{array}{r}
\left\|V_{h} \Pi u_{0, h}\right\|_{l^{5} L^{10}([0, t])} \leq\left\|V_{h}\left(\Pi u_{0, h}-v_{0, h}\right)\right\|_{l^{5} L^{10}([0, t])}+\left\|V_{h} v_{0, h}\right\|_{l^{5} L^{10}([0, t])} \\
\leq\left\|V_{h}\left(\Pi u_{0, h}-v_{0, h}\right)\right\|_{l^{5} L^{10}([0, t])}+t^{1 / 10}\left\|V_{h} v_{0, h}\right\|_{L^{\infty}[0, T] l^{\infty} \cap l^{2}} \\
\left.\lesssim \| \Pi u_{0, h}-v_{0, h}\right)\left\|_{l^{2}}+t^{1 / 10}\right\| v_{0} \|_{H^{1}}
\end{array}
$$

As limsup $\left.\| \Pi u_{0, h}-v_{0, h}\right) \|_{l^{2}} \leq \varepsilon$, by chosing $h$, (then) $t$ small enough we have obtained $h$

$$
\forall \varepsilon>0, \exists t_{0}, h_{0}>0: \forall \varepsilon \leq t \leq t_{0}, h \leq h_{0},\left\|V_{h} \Pi u_{0, h}\right\|_{l^{5} L^{10}([0, t])} \leq 2 \varepsilon,
$$

which is the expected convergence.

Remark 9. The convergence of any extracted subsequence to the solution $u$ actually proves that any sequence $P u_{h_{n}}$ converges to $u$ as $h_{n} \rightarrow 0$.

\section{Scattering}

Scattering of the solutions of the continuous $c K d V$ equation for small initial data has been known for 20 years [12]. Roughly, it means that the solution of the nonlinear equation asymptotically behaves like a solution of the linearized equation. This phenomenon is opposed to the existence of solitons, which are the canonical example of nonlinear behavior. The mathematical statement is the following.

Theorem 10. [12, Kenig-Ponce-Vega] Let $u(t)$ be the solution of $c K d V$ with initial data $u_{0}$ small enough in $L^{2}(\mathbb{R})$. Then $w(x)=u_{0}-\int_{0}^{\infty} V(-s) u^{5}(s) / 5 d$ s belongs to $L^{2}(\mathbb{R})$ and

$$
\|u(t)-V(t) w\|_{L^{2}} \rightarrow_{t \rightarrow+\infty} 0 .
$$

Remark 11. A similar result holds for $t \rightarrow-\infty$, but the functions obtained $w, \widetilde{w}$ have no reason to be equal. 
We check here as a first step that a discrete analog of the theorem above can be formulated, and then discuss the convergence of the discrete functions $w_{h}$ such that $u_{h} \sim_{t} V_{h}(t) w_{h}$ to the continuous function $w$ such that $u \sim_{t} V(t) w$.

Proposition 12. For $\left\|u_{0, h}\right\|_{l^{2}}$ satisfying the smallness condition of Theorem 6 , we denote $u_{h}$ the solution of (DcKdV). Then $w_{h}=u_{0, h}-\int_{0}^{\infty} V_{h}(-s) \Pi u_{h}^{5}(s) d s$ is in $l^{2}(\mathbb{Z})$ and is such that

$$
\left\|u_{h}(t)-V_{h}(t) w_{h}\right\|_{l^{2}} \rightarrow 0 .
$$

Proof. According to the formula

$$
u_{h}(t)=V_{h}(t) u_{0, h}-\int_{0}^{t} \partial_{h} V_{h}(t-s) \Pi E u_{h}^{5}(s) / 5 d s=V_{h}(t)\left(u_{0, h}-\int_{0}^{t} \partial_{h} V_{h}(-s) \Pi E u_{h}^{5}(s) / 5 d s\right),
$$

it is sufficient to check that $\int_{0}^{t} \partial_{h} V_{h}(-s) \Pi E u_{h}^{5}(s) d s$ converges in $l^{2}$ as $t \rightarrow \infty$, thus to check that it is a Cauchy sequence. The inequality (2.8) implies

$$
\left\|\int_{T}^{t} \partial_{h} V_{h}(-s) \Pi E u_{h}^{5}(s) d s\right\|_{l^{2}} \leq\left\|\Pi E u_{h}^{5}\right\|_{l^{1} L^{2}([T, \infty[)} \lesssim\left\|1_{t \geq T} u_{h}\right\|_{l^{5} L^{10}([T, \infty[)}^{5} .
$$

But by dominated convergence we have $\left\|1_{t \geq T} u_{h}\right\|_{l^{5} L^{10}([T, \infty[)} \rightarrow 0$, we can conclude:

$$
\left\|u_{h}(t)-V_{h}(t) w_{h}\right\|_{l^{2}} \rightarrow 0, \text { where } w_{h}=v_{0}+\int_{0}^{\infty} \partial_{h} V_{h}(-s) \Pi E u_{h}^{5}(s) d s .
$$

At this point, it should be emphasized that there is no reason for the discrete solution to behave asymptotically as the continuous one. In fact even in linear settings it is not hard to see that in general

$$
\left\|P V_{h}(t) u_{0, h}-V(t) u_{0}\right\| \nrightarrow_{t \rightarrow \infty, h \rightarrow 0} 0 .
$$

Thus we may expect at best $P w_{h} \rightarrow w$ (or equivalently $P V_{h}(-t) u_{h} \rightarrow_{t} V(-t) u$ ), this is the purpose of the following proposition.

Proposition 13. Assume that $P u_{0, h} \rightarrow_{h} u_{0} L^{2}$ and satisfies the $l^{2}$ smallness condition of Theorem [6, then $P w_{h} \rightarrow_{h} w L_{w}^{2}$.

Proof. According to the previous Proposition,

$$
w_{h}=u_{0, h}-\int_{0}^{\infty} V_{h}(-s) \partial_{h} \Pi E u_{h}^{5}(s) / 5 d s
$$

the improper integral being seen as a strong limit in $l^{2}$. Since $w=u_{0}-\int_{0}^{\infty} V(-s) \partial_{x} u^{5}(s) d s$ it is sufficient to check that

$$
\int_{0}^{\infty} P V_{h}(-s) \partial_{h} \Pi u_{h}^{5}(s) d s \rightarrow_{h} \int_{0}^{\infty} \partial_{x} V(-s) u^{5}(s) d s .
$$


Note that the convergence in $h$ of $\int_{0}^{t} V_{h}(-s) \Pi E u_{h}^{5}(s) d s$ for fixed $t$ is actually rather easy but leaves open the problem of interversion $\lim _{t} \lim _{h}=\lim _{h} \lim _{t}$. More directly we shall write

$$
\left\|\int_{0}^{\infty} P V_{h}(-s) \partial_{h} \Pi E u_{h}^{5}(s) d s\right\|_{L^{2}} \leq\left\|\Pi E u_{h}^{5}(s)\right\|_{l^{1} L^{2}} \leq\left\|u_{h}\right\| l^{5} L^{10} \leq C,
$$

so that we may extract an $L^{2}$ weakly converging subsequence. Up to further extraction we may also assume (see the proof of theorem 8 ) that $P u_{h} \rightarrow u$ a.e. . Let $\varphi$ be an $L^{2}$ function with compactly supported Fourier transform,

$$
\int_{\mathbb{R}} \int_{0}^{\infty} P \partial_{h} V_{h} \Pi E u_{h}^{5} \varphi d s d x=\int_{\mathbb{R}} \int_{0}^{T} P \partial_{h} V_{h} \Pi E u_{h}^{5} \varphi d s d x+\int_{\mathbb{R}} \int_{T}^{\infty} P \partial_{h} V_{h} \Pi E u_{h}^{5} \varphi d s d x .
$$

As a first step, we prove that the limit as $T \rightarrow \infty$ of the second term in the above right hand side is 0 uniformly in $h$. In what follows we use the fact that the Fourier multiplier $V_{h}(t)$ is formally defined by its symbol on $l^{2}(h \mathbb{Z})$ as well as on $L^{2}(\mathbb{R})$, and we have $P V_{h}=V_{h} P$. Thus we can write $\int_{\mathbb{R}} \int_{T}^{\infty} P \partial_{h} V_{h}(-t) \Pi E u_{h}^{5} \varphi d t d x=\int_{\mathbb{R}} \int_{T}^{\infty} \partial_{h} P \Pi E u_{h}^{5} V_{h}(t) \varphi d x d t$. Hölder's inequality implies

$$
\left|\int_{\mathbb{R}} \int_{T}^{\infty} \partial_{h} P \Pi E u_{h}^{5} V_{h}(t) \varphi d x d t\right| \lesssim\left\|\partial_{h} u_{h}\right\|_{l^{\infty} L^{2}([T, \infty])}\|u\|_{l^{5} L^{10}([T, \infty])}^{4}\left\|V_{h}(t) \varphi\right\|_{L^{5} L^{10}([T, \infty])} .
$$

Now using the fact that $\varphi$ has a Fourier transform compactly supported, for $h$ small enough we have $1\left\|V_{h}(t) \varphi\right\|_{L_{x}^{5} L_{t}^{10}} \lesssim\|\varphi\|_{L^{2}}$, thus

$$
\lim _{T}\left\|V_{h}(t) \varphi\right\|_{L_{x}^{5} L^{10}([T, \infty]}=0 .
$$

This implies our first step: for any $\varepsilon>0$, there exists $T_{0}>0, h_{0}>0$ such that for $T \geq$ $T_{0}, h \leq h_{0}$,

$$
\left|\int_{\mathbb{R}} \int_{T}^{\infty} P \partial_{h} V_{h} \Pi E u_{h}^{5} \varphi d s d x\right| \leq \varepsilon
$$

We now focus on the convergence of $\int_{\mathbb{R}} \int_{0}^{T} P \partial_{h} V_{h} \Pi E u_{h}^{5} \varphi d s d x$ :

$$
\begin{aligned}
\int_{\mathbb{R}} \int_{0}^{T} P \partial_{h} V_{h} \Pi E u_{h}^{5} \varphi d s d x & =\int_{\mathbb{R}} \int_{0}^{T} P \Pi E u_{h}^{5} \partial_{-h} V_{h}(-s) \varphi d s d x \\
& =\int_{\mathbb{R}} \int_{0}^{T} P \Pi E u_{h}^{5}\left(\left(V-V_{h}\right) \partial_{x} \varphi+V_{h}\left(\partial_{x}+\partial_{-h}\right) \varphi-V \partial_{x} \varphi\right) d s d x .
\end{aligned}
$$

From the proof of theorem 8 , we know that $P \Pi E u_{h}^{5} \rightarrow u^{5}\left(\mathcal{D}^{\prime}\left(\mathbb{R}^{2}\right)\right)$, and thus weakly in $L_{x}^{1} L_{t}^{2}$ since it is bounded in that space: indeed by uniqueness of the distributional limit any extracted

\footnotetext{
1 This fact may be seen as a simple corollary of the dispersive estimates proved previously: supp $\widehat{\varphi} \subset$ $[-C, C] \Rightarrow \widehat{\varphi}=\widehat{\varphi} 1_{[-\pi / 6 h, \pi / 6 h]}$ for $h$ small enough. It is thus supported away from the "bad" points $\pm 2 \pi /(3 h), \xi_{0}, \xi_{1}$.
} 
subsequence converging weakly in $L^{1} L^{2}$ has limit $u^{5}$. As a consequence

$$
\int_{\mathbb{R}} \int_{0}^{T} P \Pi E u_{h}^{5} V(s) \partial_{x} \varphi d s d x \rightarrow \int_{\mathbb{R}} \int_{0}^{T} u^{5} V(s) \partial_{x} \varphi d s d x=-\int_{\mathbb{R}} \int_{0}^{T} \partial_{x} V(-s) u^{5} \varphi d s d x .
$$

Moreover for fixed $T$, one easily sees that $\sup _{s \in[0, T]}\left\|\left(V-V_{h}\right)(-s) \partial_{x} \varphi\right\|_{H_{x}^{1}} \rightarrow 0$ (it is a simple consequence of the convergence $p_{h} \rightarrow \xi^{3}$ and the fast decrease of $\left.\widehat{\varphi}\right)$. Thus

$$
\left\|\left(V-V_{h}\right)(-s) \partial_{x} \varphi\right\|_{L_{x}^{\infty} L_{T}^{2}} \rightarrow 0, \text { and }\left\|\left(\partial_{x}+\partial_{-h}\right) \varphi\right\|_{H^{1}} \rightarrow 0,
$$

so that $\left\|V_{h}\left(\partial_{x}+\partial_{-h}\right) \varphi\right\|_{L_{x}^{\infty} L_{T}^{2}} \rightarrow 0$, and we finally get

$$
\int_{\mathbb{R}} \int_{0}^{T} P \partial_{h} V_{h}(-s) \Pi E u_{h}^{5} \varphi d s d x \rightarrow \int_{\mathbb{R}} \int_{0}^{T} u^{5} \partial_{x} V(s) \varphi d s d x=-\int_{\mathbb{R}} \int_{0}^{T} \partial_{x} V(-s) u^{5} \varphi d s d x .
$$

Using (6.2), (6.3) we find that for any $\varepsilon>0$, there exists $h_{0}$ small enough such that for $h \leq h_{0}$ :

$$
\begin{aligned}
\left|\int_{\mathbb{R}} \int_{0}^{\infty} P \partial_{h} V_{h} \Pi E u_{h}^{5} \varphi d s d x-\int_{\mathbb{R}} \int_{0}^{\infty} \partial_{x} V u^{5} \varphi d s d x\right| & \leq\left|\int_{\mathbb{R}} \int_{T}^{\infty} P \partial_{h} V_{h} \Pi E u_{h}^{5} \varphi d s d x\right| \\
+\left|\int_{\mathbb{R}} \int_{0}^{T}\left(P \partial_{h} V_{h} \Pi E u_{h}^{5}-\partial_{x} V u^{5}\right) \varphi d s d x\right| & +\left|\int_{\mathbb{R}} \int_{T}^{\infty} \partial_{x} V u^{5} \varphi d s d x\right| \\
& \leq 3 \varepsilon .
\end{aligned}
$$

We have proved that for any $\varphi$ with compactly supported Fourier transform,

$$
\int_{\mathbb{R}} \int_{0}^{\infty} P \partial_{h} V_{h} \Pi E u_{h}^{5} \varphi d s d x \rightarrow \int_{\mathbb{R}} \int_{0}^{\infty} \partial_{x} V u^{5} \varphi d s d x .
$$

But the density of these functions in $L^{2}$ implies the uniqueness of the weak $L^{2}$ limit of $u_{h}$ (if it exists), and the $L^{2}$ boundedness of $\int_{0}^{\infty} P \partial_{h} V_{h} \Pi u_{h}^{5} d t$ gives its existence by weak compacity.

\section{$7 \quad$ Rates of convergence}

Since the previous sections were only devoted to the critical case, it is very unlikely that any rate of convergence may be obtained for $L^{2}$ initial data. However if $u_{0} \in H^{s}, s>0$ the problem becomes subcritical and more quantitative estimates are expected. We first define discrete initial data converging to the continuous one in the following way. Let $T_{h}$ be defined as

$$
\begin{array}{r}
T_{h}: L^{2} \rightarrow l^{2}(h \mathbb{Z}), \\
\left(T_{h} u\right)_{n}=\frac{1}{2 \pi} \int_{-\pi / h}^{\pi / h} \widehat{u} e^{i n h \xi} d \xi,
\end{array}
$$


here we use for the usual Fourier transform the same notation $\widehat{\cdot}$ as for the discrete one. If $u_{0}$ is the initial continuous data, we simply set $u_{0, h}=T_{h} u_{0}$. By dominated convergence, it can be seen (though it is not absolutely obvious) that for any $f \in L^{2},\left\|P T_{h} f-f\right\|_{L^{2}} \rightarrow 0$, and more precisely $\left\|P T_{h} f-f\right\|_{L^{2}} \lesssim h^{s}\|f\|_{H^{s}}$. For this reason we will only study the convergence to 0 of $u_{h}-T_{h} u$ rather than $P u_{h}-u$. Note that the operator $T_{h}$ is particularly convenient since $\left.\widehat{V(t) T_{h}} u_{0}\right|_{[-\pi / h, \pi / h]}=\left.e^{i t \xi^{3} \widehat{u_{0}}}\right|_{[-\pi / h, \pi / h]}=\left.T_{h} \widehat{V(t)} u_{0}\right|_{[-\pi / h, \pi / h]}$. Consequently - as in previous section - in what follows we will abusively write $V(t)$ for both multipliers of symbol $e^{i t \xi^{3}}$ acting on $L^{2}(\mathbb{R})$ and $l^{2}(h \mathbb{Z})$.

Convergence rates were obtained for the (subcritical) nonlinear Schrödinger equation in [5] by using "discrete Littlewood-Paley analysis". Our case is slightly more complicated, for essentially three reasons; critical regularity, lack of "choice" for the Strichartz estimates, and the nonlinearity involving derivatives.

The section is divided in two parts. The first one establishes a list of linear estimates with rates of convergence. Unfortunately this list is not sufficient to obtain actual rates of convergence on $T_{h} u-u_{h}$ but we prove such results for a simpler semi-linear problem. Though it is not entirely satisfactory we believe that the result and the technics used are interesting by themselves.

\subsection{Linear estimates}

As a warm up, we first treat the control of $\left\|T_{h} V(t) u_{0}-V_{h}(t) \Pi T_{h} u_{0}\right\|_{l^{2}}$, which is quite simple but gives a good idea of the technics used in this subsection.

Proposition 14. Let $\Pi$ be an interpolator as in theorem [6, of symbol $m$ such that $m(0)=1$. For $u_{0} \in L^{2}$, we have the homogeneous estimate

$$
\left\|V(t) T_{h} u_{0}-V_{h}(t) T_{h} \Pi T_{N h} u_{0}\right\|_{L^{\infty}\left([0, T] ; l^{2}\right)} \leq C h^{2 s / 5}\left\|u_{0}\right\|_{H^{s}(\mathbb{R})} .
$$

The constant $C$ only depends on $p$ and $\Pi$.

Proof. First we note that the scheme is of order 2 in the sense that

$$
\xi^{3}-p_{h}=\xi^{3}-\frac{4 \sin ^{2}(\xi h / 2) \sin (\xi h)}{h^{3}}=O\left(h^{2} \xi^{5}\right) \text { (by Taylor expansion). }
$$

Using the inequality

$$
\forall 0 \leq \alpha \leq 1, a, b \geq 0, \min (a, b) \leq a^{\alpha} b^{1-\alpha},
$$

we have

$$
\left|e^{i t p_{h}}-e^{i t \xi^{3}}\right| \lesssim\left|t h^{2} \xi^{5}\right|^{\alpha} .
$$

We split the left hand side of (7.1) as follows

$$
\begin{aligned}
\left\|V(t) T_{h} u_{0}-V_{h}(t) \Pi T_{N h} u_{0}\right\|_{L^{\infty}\left([0, T] ; l^{2}\right)} \leq & \left\|V(t) \Pi T_{N h} u_{0}-V_{h}(t) \Pi T_{N h} u_{0}\right\|_{L^{\infty}\left([0, T] ; l^{2}\right)} \\
& +\left\|V(t)\left(T_{h}-\Pi T_{N h}\right) u_{0}\right\|_{L^{\infty}\left([0, T] ; l^{2}\right)} \\
= & N_{1}+N_{2} .
\end{aligned}
$$


$N_{1}$ is estimated thanks to (7.4):

$$
N_{1}^{2} \lesssim h^{4 s / 5} T^{2 s / 5} \int_{\mathbb{R}}|\xi|^{2 s}\left|\Pi \widehat{T_{N h} u_{0}}\right|^{2} d \xi \lesssim h^{4 s / 5}\left\|u_{0}\right\|_{H^{s}}^{2}
$$

On the other hand if we denote by $m$ the symbol of $\Pi$

$$
N_{2}^{2} \leq\left\|\left(T_{h}-\Pi T_{N h}\right) u_{0}\right\|_{\left.l^{2}\right)}=\int_{-\pi / h}^{\pi / h}\left|\widehat{u_{0}}-m(h \xi) \widetilde{u_{0}}(\xi)\right|^{2} d \xi
$$

where $\widetilde{u_{0}}$ is the $2 \pi / N h$ periodic function such that $\left.\widetilde{u_{0}}\right|_{[-\pi / N h, \pi / N h]}=\widehat{u_{0}}$. But since $m$ is bounded and $m(0)=1,|m(h \xi)-1| \lesssim h^{s}|\xi|^{s}$. We also remind (see Remark 3) that $m$ satisfies $m(2 k \pi \xi / N)=0,1 \leq k \leq N-1$, thus $m(2 k \pi / N+h \xi)=O\left(h^{s}|\xi|^{s}\right)$. This gives

$$
\begin{aligned}
\int_{-\pi / h}^{\pi / h}\left|\widehat{u_{0}}-m(h \xi) \widetilde{u_{0}}(\xi)\right|^{2} d \xi= & \int_{-\pi / N h}^{\pi / N h}\left|\widehat{u_{0}}-m(h \xi) \widehat{u_{0}}(\xi)\right|^{2} d \xi \\
& +\int_{\pi / N h \leq|\xi| \leq \pi / h}\left|\widehat{u_{0}}-m(h \xi) \widetilde{u_{0}}(\xi)\right|^{2} d \xi \\
\lesssim & \int_{-\pi / h}^{\pi / h} h|\xi|^{2}\left|\widehat{u_{0}}\right|^{2} d \xi+\left.\sum_{k=1}^{N-1} \int_{(2 k-1) \pi / N h}^{(2 k+1) \pi / N h}|m(h \xi)| \widehat{u_{0}}(\xi)\right|^{2} d \xi . \\
\lesssim & h^{2 s} \int_{-\pi / N h}^{\pi / N h}|\xi|^{2 s}\left|\widehat{u_{0}}\right|^{2} d \xi+h^{2 s} \int_{-\pi / h}^{\pi / h}|\xi|^{2 s}\left|\widehat{u_{0}}\right|^{2} d \xi \\
\leq & 2 h^{2 s}\left\|u_{0}\right\|_{H^{s}}^{2} .
\end{aligned}
$$

Summing (7.5), (17.6) we get

$$
\left\|V(t) T_{h} u_{0}-V_{h}(t) \Pi T_{N h} u_{0}\right\|_{L^{\infty}\left([0, T] ; l^{2}\right)} \leq C\left(1+T^{s / 5}\right)\left(h^{2 s / 5}+h^{s / 2}\right)\left\|u_{0}\right\|_{H^{s}} \lesssim h^{2 s / 5}\left\|u_{0}\right\|_{H^{s}} .
$$

Proposition 15. Under the same assumptions as Prop. 14, and for any $g \in l^{4 / 3} L_{T}^{1}$,

$$
\begin{array}{r}
\left\||D|^{-1 / 4}\left(V(t) T_{h} u_{0}-V_{h}(t) \Pi T_{N h}\right) u_{0}\right\|_{l^{4} L^{\infty}([0, T])} \leq C(T) h^{2 s / 5}\left\|u_{0}\right\|_{H^{s}(\mathbb{R})}, \\
\left\||D|^{-1 / 2} \int_{0}^{t}\left(V(t-s) g-V_{h}(t-s)\right) \Pi T_{N h} g\right\|_{l^{4} L_{T}^{\infty}} \leq C(T) h^{2 s / 5}\left\||D|^{s} \Pi T_{N h} g\right\|_{l^{4 / 3} L^{1}([0, T])} .
\end{array}
$$

Proof. As in the proof of Prop 14, we write

$$
\begin{aligned}
\left\||D|^{-1 / 4}\left(V(t) T_{h} u_{0}-V_{h}(t) \Pi T_{N h} u_{0}\right)\right\|_{l^{4} L^{\infty}([0, T])} & \leq\left\||D|^{-1 / 4}\left(V-V_{h}\right) \Pi T_{N h} u_{0}\right\|_{l^{4} L^{\infty}}(7.9) \\
& +\left\||D|^{-1 / 4} V\left(T_{h}-\Pi T_{N h}\right) u_{0}\right\|_{l^{4} L^{\infty}(.7 .10)}
\end{aligned}
$$


We have directly using (7.6)

$$
\left\||D|^{-1 / 4} V(t)\left(T_{h}-\Pi T_{N h}\right) u_{0}\right\|_{l^{4} L^{\infty}([0, T])} \lesssim\left\|\left(T_{h}-\Pi T_{N h}\right) u_{0}\right\|_{l^{2}} \lesssim h^{s}\left\|u_{0}\right\|_{H^{s}},
$$

so that it suffices to prove the (more precise) estimate

$$
\left\|V(t) \Pi T_{N h} u_{0}-V_{h}(t) \Pi T_{N h} u_{0}\right\|_{l^{4} L^{\infty}([0, T])} \lesssim h^{2 s / 5}(1+T)^{s / 5}\left\|\left|D_{x}\right|^{s} \Pi T_{N h} u_{0}\right\|_{L^{2}} .
$$

A careful look at the proof of Prop 4 shows that it amounts to the estimate

$$
\left|\int_{-\pi / h}^{\pi / h} \psi_{h} \frac{e^{i t p_{h}(\eta)}-e^{i t \eta^{3}}}{|\eta|^{1 / 2+s}} e^{i j h \eta} d \eta\right| \leq C \frac{h^{2 s / 5}(1+T)^{s / 5}}{(h(1+|j|))^{1 / 2}}
$$

where $\psi_{h}$ is the symbol of $\Pi$ and cancels at $\pm 2 \pi /(3 h), \xi_{0} / h, \xi_{1} / h$. Since (7.12) also implies by the duality argument of Prop 4 the estimate (7.8), the rest of the proof is devoted to its derivation. Set $\tau=t / h^{3}$, after the change of variable $\eta=\xi / h$, (7.11) is equivalent to

$$
\left|\int_{-\pi}^{\pi} \psi(\xi) \frac{e^{i \tau p(\xi)}-e^{i \tau \xi^{3}}}{|\xi|^{1 / 2+s}} e^{i j \xi} d \xi\right| \leq C \frac{\tau^{s / 5}}{(1+|j|)^{1 / 2}} .
$$

By parity, we may may reduce it to

$$
\left|\int_{0}^{\pi} \psi(\xi) \frac{e^{i \tau p(\xi)}-e^{i \tau \xi^{3}}}{|\xi|^{1 / 2+s}} e^{i j \xi} d \xi\right| \leq C \frac{\tau^{s / 5}}{(1+|j|)^{1 / 2}} .
$$

The proof of this estimate is rather delicate, in fact it follows the proof of Prop 4 with some non trivial modifications. Since a lot of quantities which will appear are estimated by similar technics, we will often skip details.

We will use repeatedly the fact that $\xi$ lies in a bounded set, thus the inequality $\left|p-\xi^{3}\right| \lesssim|\xi|^{5}$ implies $\left|p-\xi^{3}\right| \lesssim|\xi|^{r}$ for $0 \leq r \leq 5$. Similarly $\left|e^{i \tau p}-e^{i \tau \xi^{3}}\right| \leq\left|\tau \xi^{5}\right|^{r}$ for $0 \leq r \leq 1$. First note that for $j=0$ the result is trivial since

$$
\left|\int_{0}^{\pi} \psi(\xi) \frac{e^{i \tau p(\xi)}-e^{i \tau \xi^{3}}}{|\xi|^{1 / 2+s}} e^{i j \xi} d \xi\right| \lesssim \int_{0}^{\pi} \frac{\tau^{s / 5}|\xi|^{s}}{|\xi|^{1 / 2+s}} d \xi \lesssim \tau^{s / 5}
$$

We split $[0, \pi]$ as

$$
\begin{array}{r}
A_{1}=\left[0, \tau^{-1 / 3}\right] \cap[0, \pi], \\
A_{2}=A_{1}^{c} \cap\left\{\min \left(\left|3 \tau \xi^{2}+j\right|,\left|\tau p^{\prime}(\xi)+j\right|\right) \geq|j| / 2\right\}, \\
A_{3}=\left(A_{1} \cup A_{2}\right)^{c} .
\end{array}
$$

Let us fix $C$ large enough such that

$$
\tau^{-1 / 3} \geq C /|j| \Rightarrow \forall \xi \in\left[0, \tau^{-1 / 3}\right], \min \left(\left|j+3 \tau \xi^{2}\right|,\left|j+\tau p^{\prime}\right|\right) \geq|j| / 2 .
$$


If $\tau^{-1 / 3} \leq C /|j|$

$$
\left|\int_{A_{1}} \psi(\xi) \frac{e^{i \tau p(\xi)}-e^{i \tau \xi^{3}}}{|\xi|^{1 / 2+s}} e^{i j h \xi} d \xi\right| \leq \int_{0}^{C /|j|} \frac{\tau^{s / 5}}{|\xi|^{1 / 2}} d \xi \lesssim \frac{\tau^{s / 5}}{|j|^{1 / 2}}
$$

and the estimate on $A_{1}$ is complete. Else

$$
\begin{aligned}
\left|\int_{A_{1}} \psi(\xi) \frac{e^{i \tau p(\xi)}-e^{i \tau \xi^{3}}}{|\xi|^{1 / 2+s}} e^{i j \xi} d \xi\right| \leq & \left|\int_{0}^{C /|j|} \psi(\xi) \frac{e^{i \tau p(\xi)}-e^{i \tau \xi^{3}}}{|\xi|^{1 / 2+s}} e^{i j \xi} d \xi\right| \\
& +\left|\int_{C /|j| \leq \xi \leq \tau^{-1 / 3}} \psi(\xi) \frac{e^{i \tau p(\xi)}-e^{i \tau \xi^{3}}}{|\xi|^{1 / 2+s}} e^{i j \xi} d \xi\right| \\
\lesssim & \left|\int_{C /|j| \leq \xi \leq \tau^{-1 / 3}} \psi(\xi) \frac{e^{i t / h^{3} p(\xi)}-e^{i t / h^{3} \xi^{3}}}{|\xi|^{1 / 2+s}} e^{i j \xi} d \xi\right| \\
& +\frac{\tau^{s / 5}}{|j|^{1 / 2}} .
\end{aligned}
$$

On $C /|j| \leq \xi \leq \tau^{-1 / 3}$, an integration by part gives

$$
\begin{aligned}
\int_{C /|j|}^{\tau^{-1 / 3}} \psi(\xi) \frac{e^{i \tau p(\xi)}-e^{i \tau \xi^{3}}}{|\xi|^{1 / 2+s}} e^{i j \xi} d \xi & =\frac{e^{i j \tau^{-1 / 3}} \psi\left(\tau^{-1 / 3}\right)}{\left|\tau^{-1 / 3}\right|^{1 / 2+s}}\left(\frac{e^{i \tau^{3} p\left(\tau^{-1 / 3}\right)}}{j+\tau p^{\prime}\left(\tau^{-1 / 3}\right)}-\frac{e^{i}}{j+3 \tau^{1 / 3}}\right) \\
& -j^{1 / 2+s} \psi(C /|j|) e^{i \operatorname{sgn}(j)}\left(\frac{e^{i \tau p(C /|j|)}}{j+\tau p^{\prime}(C /|j|)}-\frac{e^{i \tau(C / j)^{3}}}{j+\frac{3 C^{2} \tau}{j^{2}}}\right) \\
& +\int_{C /|j|}^{\tau^{-1 / 3}} e^{i \tau \xi^{3}}\left(\frac{\psi}{\left(j+3 \tau \xi^{2}\right)|\xi|^{1 / 2+s}}\right)^{\prime} d \xi \\
& -\int_{C /|j|}^{\tau^{-1 / 3}} e^{i \tau p}\left(\frac{\psi}{\left(j+\tau p^{\prime}\right)|\xi|^{1 / 2+s}}\right)^{\prime} d \xi .
\end{aligned}
$$

Remind that $\tau$ satisfies (7.13). The first term is estimated using

$$
e^{i \tau p\left(\tau^{-1 / 3}\right)}-e^{i \tau\left(\tau^{-1 / 3}\right)^{3}}=O\left(\tau^{-2 / 3}\right), 3 \tau^{-2 / 3}-p^{\prime}\left(\tau^{-1 / 3}\right)=O\left(\tau^{-4 / 3}\right),
$$

which gives

$$
\begin{array}{r}
\left|\tau^{(1 / 2+s) / 3}\left(\frac{e^{i \tau p\left(\tau^{-1 / 3}\right)}}{j+\tau p^{\prime}\left(\tau^{-1 / 3}\right)}-\frac{e^{i}}{j+3 \tau^{1 / 3}}\right)\right|=\left|\tau^{1 / 3(1 / 2+s)}\left(\frac{e^{i \tau p\left(\tau^{-1 / 3}\right)}}{j+\tau p^{\prime}\left(\tau^{-1 / 3}\right)}-\frac{e^{i}}{j+3 \tau^{1 / 3}}\right)\right| \\
=\tau^{(1 / 2+s) / 3} \frac{e^{i \tau p\left(\tau^{-1 / 3}\right)}\left(j+3 \tau^{1 / 3}\right)-e^{i}\left(j+\tau p^{\prime}\left(\tau^{-1 / 3}\right)\right)}{\left(j+\tau p^{\prime}\left(\tau^{-1 / 3}\right)\left(j+3 \tau^{-1 / 3}\right)\right.} \\
=\tau^{(1 / 2+s) / 3}\left|\frac{j\left(e^{i \tau p}-e^{i}\right)+\tau^{1 / 3}\left(3 e^{i \tau p}-3 e^{i}+3 e^{i}-\tau^{2 / 3} p^{\prime} e^{i}\right)}{\left(j+\tau p^{\prime}\right)\left(j+3 \tau^{-1 / 3}\right)}\right| \\
\lesssim \tau^{(1 / 2+s) / 3} \frac{\left|j \tau^{-2 s / 15}\right|}{j^{2}} \cdot \lesssim \frac{\tau^{s / 5}}{\sqrt{j}}
\end{array}
$$


The estimate for the second term is similar, we give details for two of the remaining integral terms involved:

$$
\begin{gathered}
\left|\int_{C /|j|}^{\tau^{-1 / 3}} e^{i \tau \xi^{3}} \frac{6 \tau \xi \psi(\xi)}{\left.\left(j+3 \tau \xi^{2}\right)^{2} \xi^{1 / 2+s}\right)}-e^{i \tau p} \frac{\tau p^{\prime \prime}(\xi) \psi(\xi)}{\left(j+\tau p^{\prime}(\xi)\right) \xi^{1 / 2+s}} d \xi\right| \\
\lesssim \int_{C /|j|}^{\tau^{-1 / 3}} \frac{\tau\left|p^{\prime \prime}-6 \xi\right|}{j^{2} \xi^{1 / 2+s}} d \xi+\int_{C /|j|}^{\tau^{-1 / 3}} \frac{\tau\left|p^{\prime \prime}\right|}{\xi^{1 / 2+s}}\left|\frac{1}{\left(j+\tau p^{\prime}\right)^{2}}-\frac{1}{\left(j+3 \tau \xi^{2}\right)^{2}}\right| \\
\lesssim \int_{C /|j|}^{\tau^{-1 / 3}} \frac{\tau^{s / 5} \xi^{1+2 s / 5}}{j^{2} \xi^{7 / 2+2 s / 5}} d \xi+\int_{C /|j|}^{\tau^{-1 / 3}} \frac{\tau\left|p^{\prime \prime}\right|}{\xi^{1 / 2+s} j^{4}}\left|2 j \tau\left(3 \xi^{2}-p^{\prime}(\xi)\right)+9 \xi^{4} \tau^{2}-\left(p^{\prime}\right)^{2} \tau^{2}\right| \\
\lesssim \frac{\tau^{s / 5}}{\sqrt{|j|}}+\int_{C /|j|}^{\tau^{-1 / 3}} \frac{\tau|\xi|}{\xi^{1 / 2+s} j^{4}}\left(\left|2 j \tau \xi^{2+2 s / 5}\right|+\tau^{2} \xi^{4+2 s / 5}\right) d \xi \\
\left.\lesssim \frac{\tau^{s / 5}}{\sqrt{|j|}}+\int_{C /|j|}^{\tau^{-1 / 3}} \frac{\tau^{s / 5}}{\xi^{3+1 / 2}|j|^{3}}+\frac{\tau^{s / 5}}{\xi^{4+1 / 2} j^{4}}\right) d \xi \\
\lesssim \frac{\tau^{s / 5}}{\sqrt{|j|}}
\end{gathered}
$$

The analysis on $A_{2}$ is similar (and in fact simpler) so we skip it and prove the estimate for the integral on $A_{3}=\left\{\left|\tau^{-1 / 3}\right| \leq|\xi| \leq \pi\right\} \cap\left\{\min \left(\left.|j+3 \tau| \xi\right|^{2}|| j+,\tau p^{\prime}(\xi) \mid\right) \leq|j| / 2\right\}$.

Since $1 / \xi^{1 / 2+s}$ is a smooth function away from 0 , for any fixed $\varepsilon>0$ we may directly carry the proof of proposition 4 to obtain

$$
\left|\int_{A_{3} \cap\{\xi \geq \varepsilon\}} \psi(\xi) \frac{e^{i \tau p(\xi)}-e^{i \tau \xi^{3}}}{|\xi|^{1 / 2+s}} e^{i j \xi} d \xi\right| \leq \frac{C(\varepsilon)}{|j|^{1 / 2}} \leq \frac{C(\varepsilon)(1+\tau)^{s / 5}}{|j|^{1 / 2}} .
$$

Thus we only have to treat the integral on $A_{4}:=A_{3} \cap\{\xi \leq \varepsilon\}$, and in particular if $\varepsilon$ is small enough we may assume the following

- for $\xi \in A_{4},|\xi| \asymp \sqrt{|j| / \tau} \ll 1$,

- $A_{4}$ is an interval of measure dominated by $\sqrt{|j| / \tau}$,

- The equation $j+\tau p^{\prime}(\xi)=0$ has an unique root $\xi_{2}$ such that $\xi_{2} \asymp \sqrt{-j / \tau}$.

We define $f(\xi)=e^{i j \xi}\left(e^{i \tau \xi^{3}}-e^{i \tau p(\xi)}\right)$. Let $\xi_{1}$, be the positive solution of $j+3 \tau \xi^{2}=0$. Clearly $\xi_{1} \leq \xi_{2}$ and we define $F(\xi)=\int_{\xi_{1}}^{\xi} f(\eta) d \eta$. An integration by parts gives

$$
\begin{aligned}
\left|\int_{A_{4}} f(\xi) \frac{\psi(\xi)}{\xi^{1 / 2+s}} d \xi\right| & \leq\left|\left[F \frac{\psi}{\xi^{1 / 2+s}}\right]_{\min A_{4}}^{\max A_{4}}\right|+\left|\int_{A_{4}} F(\xi)\left(\frac{\psi^{\prime}}{\xi^{1 / 2+s}}-\frac{(1 / 2+s) \psi}{\xi^{3 / 2+s}}\right) d \xi\right| \\
& \lesssim\|F\|_{L^{\infty}\left(A_{4}\right)}\left\|\xi^{-1 / 2-s}\right\|_{L^{\infty}\left(A_{4}\right)} \\
& \lesssim\|F\|_{L^{\infty}(\tau /|j|)^{1 / 4+s / 2}}
\end{aligned}
$$


In view of the above inequality, we are reduced to prove $\|F\|_{\infty} \lesssim \frac{|j|^{s / 2} \tau^{-3 s / 10}}{|j \tau|^{1 / 4}}$. Firstly, by application of the Van der Corput lemma :

$$
\|F\|_{L^{\infty}\left(A_{4}\right)} \lesssim \frac{1}{\sqrt{\tau \inf _{A_{4}} \min \left(p^{\prime \prime}, 6 \xi\right)}} \lesssim \frac{1}{|j \tau|^{1 / 4}},
$$

which rules out the case $|j|^{s / 2} \tau^{-3 s / 10} \geq 1$. Moreover if $\tau \geq|j|^{3}$ we have $A_{4} \subset A_{1}$ thus we can assume without loss of generality

$$
|j|^{3} \geq \tau \geq|j|^{5 / 3}
$$

We will repeatedly use that for $\xi \asymp \sqrt{|j| / \tau \mid}, 0 \leq \alpha \leq 1 / 2$,

$$
|f(\xi)| \lesssim\left|\tau \xi^{5}\right|^{2 \alpha} \lesssim|j|^{5 \alpha} \tau^{-3 \alpha} .
$$

A basic asymptotic development shows that $\left|\xi_{2}-\xi_{1}\right|=O\left(\sqrt{|j| / \tau}^{3}\right)=O(1 /|j|)$ thus (7.15) implies

$$
\left|\int_{\xi_{1}}^{\xi_{2}} f d \eta\right| \lesssim \frac{|j|^{s / 2+5 / 12} \tau^{-3 s / 10-1 / 4}}{|j|} \leq \frac{|j|^{s / 2} \tau^{-3 s / 10}}{(|j| \tau)^{1 / 4}}
$$

It remains to prove

$$
\begin{aligned}
& \forall \xi \in A_{4}, \xi \geq \xi_{2},\left|\int_{\xi_{2}}^{\xi} f d \eta\right| \leq \frac{|j|^{s / 2} \tau^{-3 s / 10}}{|j \tau|^{1 / 4}}, \\
& \forall \xi \in A_{4}, \xi \leq \xi_{1},\left|\int_{\xi_{1}}^{\xi} f d \eta\right| \leq \frac{|j|^{s / 2} \tau^{-3 s / 10}}{|j \tau|^{1 / 4}}
\end{aligned}
$$

We detail the estimate (17.16), the other is similar : let us set $\delta=|j \tau|^{-1 / 4}(\leq \sqrt{|j| / \tau}$ by (17.14) $)$, and split

$$
\begin{aligned}
\left|\int_{\xi_{2}}^{\xi} f(\eta) d \eta\right| \leq & \left|\int_{\xi_{2}}^{\xi_{2}+\delta} f(\eta) d \eta\right|+\left|\int_{\xi_{2}+\delta}^{\xi} f(\eta) d \eta\right| \\
\lesssim & \frac{\tau^{-3 s / 5}|j|^{s / 2}}{|j \tau|^{1 / 4}}+\left|\left[\frac{e^{i \tau \eta^{3}+i j \xi}}{j+3 \tau \eta^{2}}-\frac{e^{i \tau p+i j \xi}}{j+\tau p^{\prime}}\right]_{\xi_{2}+\delta}^{\xi}\right| \\
& +\left|\int_{\xi_{2}+\delta}^{\xi} \frac{e^{i \tau \eta^{3}+i j \xi} 6 \tau \eta}{\left(j+3 \tau \eta^{2}\right)^{2}}-\frac{e^{i \tau p+i j \xi} \tau p^{\prime \prime}}{\left(j+\tau p^{\prime}\right)^{2}} d \eta\right|
\end{aligned}
$$

After a few basic calculations, we are reduced to estimate a (large) number of terms which are all similar to one of the following :

$$
e^{i \tau\left(\xi_{2}+\delta\right)^{3}} \tau \frac{p^{\prime}\left(\xi_{2}+\delta\right)-3\left(\xi_{2}+\delta\right)^{2}}{\left(j+3 \tau\left(\xi_{2}+\delta\right)^{2}\right)^{2}}, \frac{e^{i \tau\left(\xi_{2}+\delta\right)^{3}}-e^{i \tau p\left(\xi_{2}+\delta\right)}}{j+3 \tau\left(\xi_{2}+\delta\right)^{2}}, \int_{\xi_{2}+\delta}^{\xi} e^{i \tau \eta^{3}+i j \eta} \tau \frac{p^{\prime \prime}(\eta)-6 \eta}{\left(j+3 \tau \eta^{2}\right)^{2}} d \eta
$$


On $A_{4},|\xi| \asymp \sqrt{|j| / \tau}$, thus $\left|j+3 \tau \xi^{2}\right| \asymp\left|\xi-\xi_{1}\right| \sqrt{|j| \tau}$. Combining this with (17.14) gives for the first term

$$
\begin{aligned}
& \left|e^{i \tau\left(\xi_{2}+\delta\right)^{3}} \tau \frac{p^{\prime}\left(\xi_{2}+\delta\right)-3\left(\xi_{2}+\delta\right)^{2}}{\left(j+3 \tau\left(\xi_{2}+\delta\right)^{2}\right)^{2}}\right| \lesssim \frac{\tau\left|\xi_{2}+\delta\right|^{3}}{\left(\sqrt{|j \tau| \mid} \xi_{2}+\delta-\xi_{1} \mid\right)^{2}} \lesssim \sqrt{\frac{\tau}{|j|}} \sqrt{\frac{|j|}{\tau}}^{3} \\
& =\frac{|j|^{s / 2} \tau^{-3 s / 10}}{|j \tau|^{1 / 4}} \frac{|j|^{3 / 2-1 / 4-s / 2}}{\tau^{3 / 4-3 s / 10}} \\
& \lesssim \frac{|j|^{s / 2} \tau^{-3 s / 10}}{|j \tau|^{1 / 4}}
\end{aligned}
$$

The second term is simpler, indeed $\left|j+3 \tau\left(\xi_{2}+\delta\right)^{2}\right| \gtrsim|j \tau|^{1 / 4}$ thus (17.15) with $\alpha=s / 10$ gives

$$
\left|\frac{e^{i \tau\left(\xi_{2}+\delta\right)^{3}}-e^{i \tau p\left(\xi_{2}+\delta\right)}}{j+3 \tau\left(\xi_{2}+\delta\right)^{2}}\right| \lesssim \frac{|j|^{s / 2} \tau^{-3 s / 10}}{|j \tau|^{1 / 4}} .
$$

Finally the third term is estimated like the first one :

$$
\left|\int_{\xi_{2}+\delta}^{\xi} e^{i \tau \eta^{3}+i j \eta} \tau \frac{p^{\prime \prime}(\eta)-6 \eta}{\left(j+3 \tau \eta^{2}\right)^{2}} d \eta\right| \lesssim \frac{\tau(|j| / \tau)^{3 / 2}}{(\sqrt{j \tau} \delta)^{2}}=\sqrt{\frac{\tau}{|j|}} \sqrt[\sqrt{\frac{|j|}{\tau}}^{3}]{\frac{|j|^{s / 2} \tau^{-3 s / 10}}{|j \tau|^{1 / 4}}} .
$$

This ends the proof of (7.16).

Remark 16. So far in every estimates one looses $s$ derivatives to gain a rate in $h^{2 s / 5}$. This is probably optimal since it has the scaling of the inequality $\left|p-p_{h}\right| \leq C h^{2}|\xi|^{5}$. To the contrary, the $l^{5} L^{10}$ estimate will not be optimal, this is due to the fact that it is obtained via the interpolation of the estimates above with the dispersive smoothing results of section 2 .

Nevertheless, it should be noticed that without dispersive estimates, one may only obtain for example

$$
\left\|\left(V(t)-V_{h}(t)\right) \partial_{h} \Delta_{j} \Pi T_{N h} u_{0}\right\|_{l^{\infty} L_{T}^{2}} \leq h^{2 s / 5} 2^{j(s+3 / 2)}\left\|\Delta_{j} \Pi T_{N h} u_{0}\right\|_{l^{2}},
$$

this would lead to estimates involving $h^{2 s / 5}\left\|u_{0}\right\|_{H^{3 / 2+s}}$ putting low regularity results out of reach.

Using the interpolation argument of [13] prop. 7.4 (as for the Corollary 2 ) we have the following.

Proposition 17. Let $u_{0} \in L^{2}(\mathbb{R}), g \in l^{5 / 4}\left(h \mathbb{Z} ; L_{T}^{10 / 9}\right)$, we have the following estimates

$$
\begin{array}{r}
\left\|V(t) T_{h} u_{0}-V_{h}(t) \Pi T_{N h} u_{0}\right\|_{l^{5} L_{T}^{10}} \leq C(T) h^{8 s / 25}\left\|u_{0}\right\|_{H^{s}(\mathbb{R})}, \\
\left\|\int_{0}^{t} V(t-s) g-V_{h}(t-s) \Pi T_{N h} g\right\|_{l^{5} L_{T}^{10}} \leq C(T) h^{8 s / 25}\left\||D|^{s} T_{h} g\right\|_{l^{5 / 4} L^{10 / 9}(([0, T])} .
\end{array}
$$

Remark 18 . The exponent $8 / 25$ comes from the weights in the interpolation, which are respectively $4 / 5$ for inequality (7.7) and $1 / 5$ for inequality (2.3).

More dispersive estimates with rates (not useful for the next subsection) are given in Appendix B. 


\subsection{A simpler problem}

Though we did not manage to collect enough dispersive estimates in order to obtain rates of convergence for the approximation of the $\mathrm{cKdV}$ problem, we will describe for a simpler problem how these estimates may be succesfully used. Let us consider the semi-linear equation

$$
\left\{\begin{array}{l}
\partial_{t} u+\partial_{x}^{3} u+f(u)=0 \\
\left.u\right|_{t=0}=u_{0} \in L^{2}
\end{array}\right.
$$

where $f(u)=u|u|^{3 / 2}$. It is quite clear that the existence of an $L^{2}$ solution may not be obtained by basic semigroup methods, however using $\|V(t) u\|_{L_{x}^{5} L_{t}^{10}} \lesssim\left\|u_{0}\right\|_{L^{2}}$ and its homogeneous counterpart, we can solve the equation

$$
T u=u \text { where } T u(t)=V(t) u_{0}-\int_{0}^{t} V(t-s) f(u)(s) d s
$$

by a fixed point argument for small times or small initial data. Indeed

$$
\begin{array}{r}
\|T u\|_{L_{x}^{2}} \leq\left\|u_{0}\right\|_{L^{2}}+\|V(t-\cdot) f(u)\|_{L_{x}^{2} L_{t}^{1}} \leq\left\|u_{0}\right\|_{L^{2}} \\
\leq t^{1 / 2}\|V(t-\cdot) f(u)\|_{L_{x}^{2} L_{t}^{2}} \\
\leq\left\|u_{0}\right\|_{L^{2}}+t^{1 / 2}\|f(u)\|_{L_{x}^{2} L_{t}^{2}} \\
\leq\left\|u_{0}\right\|_{L^{2}}+t^{1 / 2}\|u\|_{L_{x}^{5} L_{t}^{5}}^{5 / 2} \\
\leq\left\|u_{0}\right\|_{L^{2}}+t^{3 / 4}\|u\|_{L_{x}^{5} L_{t}^{10}}^{5 / 2}
\end{array}
$$

similarly

$$
\begin{aligned}
\|T u\|_{L_{x}^{5} L_{t}^{10}} \leq\left\|u_{0}\right\|_{L^{2}}+\|u\|_{L_{x}^{25 / 8} L_{t}^{25 / 9}}^{5 / 2} & \leq\left\|u_{0}\right\|_{L^{2}}+t^{1 / 10}\|u\|_{L_{x, t}^{25 / 8}}^{5 / 2} \\
& \lesssim\left\|u_{0}\right\|_{L^{2}}+t^{1 / 10}\left(\|u\|_{L_{t}^{25 / 8} L_{x}^{2}}^{5 / 2}+\|u\|_{L_{t}^{25 / 8} L_{x}^{5}}^{5 / 2}\right) \\
& \leq\left\|u_{0}\right\|_{L^{2}}+t^{13 / 20}\|u\|_{L_{x}^{5} L_{t}^{10}}^{5 / 2}+t^{9 / 10}\|u\|_{L_{t}^{\infty} L_{x}^{2}}^{5 / 2}
\end{aligned}
$$

For $t$ small enough (or small initial data), these estimates are sufficient to apply the PicardBanach fixed point theorem in the space $X_{T}=L_{T}^{\infty} L_{x}^{2} \cap L_{x}^{5} L_{T}^{10}$, which implies existence and uniqueness of a solution in this space.

We focus now on the derivation of rates of convergence. We define as for $(c K d V)$ the semidiscrete approximation scheme

$$
\left\{\begin{array}{l}
\frac{d}{d t} u_{n}+\frac{u_{n+2}-2 u_{n+1}+2 u_{n-1}-u_{n-2}}{h^{3}}+\left(\Pi E_{N h} f\left(u_{h}\right)\right)_{n}=0, \\
\left.u_{h}\right|_{t=0}=\Pi T_{N h} u_{0},
\end{array}\right.
$$

Using the discrete version of $X_{T}, X_{h, T}=L_{T}^{\infty} l_{x}^{2} \cap l_{x}^{5} L_{T}^{10}$, it can be proved as for the continuous problem that for $T$ small enough there exists an unique solution $u_{h}$ of this problem admitting bounds in $X_{h, T}$ independent of $h$. The following theorem establishes a precise convergence of $u_{h}$ to $u$ as $h \rightarrow 0$. 
Theorem 19. Let $u$ be the solution of (7.20) and $u_{h}$ the solution of (7.21). For $T$ small enough and $0<s \leq 1$

$$
\left\|u_{h}-T_{h} u\right\|_{X_{h, T}} \lesssim h^{8 s / 25}\left(\|u\|_{X_{T}}+\left\||D|^{s} u\right\|_{X, T}+\|u\|_{X_{T}}^{5 / 2}+\left\||D|^{s} u\right\|_{X_{T}}^{5 / 2}\right) .
$$

The $W^{s, p}$ spaces are defined here as the usual Bessel potential spaces, namely

$$
\left\{f: \mathcal{F}^{-1}\left((1+|\xi|)^{s} \widehat{f}\right) \in L^{p}\right\} .
$$

For the proof of the theorem we will need several technical properties on fractional derivation and Fourier multipliers:

- Fractional chain rule : for any $\alpha \in(0,1), p, p_{1}, p_{2}$ such that $\frac{1}{p}=\frac{1}{p_{1}}+\frac{1}{p_{2}}$, for $F$ differentiable $\left\||D|^{\alpha} F(f)\right\|_{L^{p}} \leq C\left\|F^{\prime}(f)\right\|_{L^{p_{1}}}\left\||D|^{\alpha} f\right\|_{L^{p_{2}}}$ (see [1] section 3 for a proof).

- The $l^{p}$ norm of $T_{h} f$ is equivalent to the $L^{p}$ norm of $\mathcal{F}^{-1}\left(\chi_{[-\pi / h, \pi / h]} \widehat{f}\right)$, independently of $h$ (see Lemma 2.1 in [5], referring itself to the classical article [15], we include a sketch of proof for the estimate $\left\|\mathcal{F}^{-1} T_{h} f\right\|_{L^{p}} \leq\left\|T_{h} f\right\|_{l^{p}}$ in the appendix).

- We have for any $1<p<\infty$,

$$
\left\|T_{h} f-\Pi T_{N h} f\right\|_{l^{p}} \leq C h^{s}\left\||D|^{s} f\right\|_{L^{p}(\mathbb{R})} .
$$

This is proved for a slightly less general $\Pi$ in $[5]$ in the end of the proof of Theorem 4.2. It relies on their Lemma 2.1 combined with a Marcinkiewicz multiplier theorem (that they state in appendix). The main ingredient is that the symbol of $T_{h}-\Pi T_{N h}$ is bounded by $h^{s}|\xi|^{s}$.

- For $s \in(0,1)$, there exists $C>0$ independent of $h$ such that

$$
\left\|f\left(T_{h} u\right)-T_{h} f(u)\right\|_{l^{2}} \leq C h^{s}\|u\|_{W^{s, 5}}^{5 / 2},\left\|f\left(T_{h} u\right)-T_{h} f(u)\right\|_{l^{5 / 4}} \leq C h^{s}\|u\|_{W^{s, 25 / 8}}^{5 / 2},
$$

again, the proof is given in [5], Lemma 5.2, for integration exponents different of $(2,5)$, but the proof can be adapted without significant modifications.

Proof. We begin by writing the difference as

$$
u_{h}-T_{h} u=V(t) T_{h} u_{0}-V_{h}(t) \Pi T_{N h} u_{0}-\int_{0}^{t} V(t-s) T_{h} f(u)(s)-V_{h}(t-s) \Pi E f\left(u_{h}\right)(s) d s .
$$

The linear term is directly controlled by applying inequalities (7.18) and (7.1) :

$$
\left\|V(t) T_{h} u_{0}-V_{h}(t) \Pi T_{N h} u_{0}\right\|_{X_{h, T}} \leq C(T) h^{8 s / 25}\left\|u_{0}\right\|_{H^{s}(\mathbb{R})} .
$$


We split the non-linear term as follows :

$$
\begin{array}{r}
\int_{0}^{t} V(t-s) T_{h} f(u)(s)-V_{h}(t-s) \Pi f\left(u_{h}\right)(s) d s=\int_{0}^{t} V(t-s)\left(T_{h} f(u)-\Pi T_{N h} f(u)\right) \\
+\left(V-V_{h}\right) \Pi T_{N h} f(u)+V_{h} \Pi\left(T_{N h}-E T_{h}\right) f(u)+V_{h} \Pi E\left(T_{h} f(u)-f\left(u_{h}\right)\right) d s \\
=I_{1}+I_{2}+I_{3}+I_{4} .
\end{array}
$$

Since most of the estimates are obtained in very similar ways, we will only detail how to deal with $I_{1}$ and $I_{4}$. The $L^{2}$ bound for $I_{1}$ is obtained by using (7.6) and the fractional chain rule with $p_{1}=10 / 3, p_{2}=5$ :

$$
\begin{aligned}
&\left\|\int_{0}^{t} V(t-s)\left(T_{h} f(u)-\Pi T_{N h} f(u)\right) d s\right\|_{L^{2}} \lesssim t^{1 / 2} \| T_{h} f(u)\left.-\Pi T_{N h} f(u)\right) d s \|_{L_{T}^{2} l^{2}} \\
& \lesssim h^{s}\left\||D|^{s} f(u)\right\|_{L_{T}^{2} L_{x}^{2}} \\
& \lesssim h^{s}\|u\|_{L_{x}^{5} L_{T}^{10}}^{3 / 2}\left\||D|^{s} u\right\|_{L_{x}^{5} L_{T}^{10}}
\end{aligned}
$$

For the $l^{5} L_{T}^{10}$ bound, we use the estimate (7.23) to get

$$
\begin{aligned}
\left\|\int_{0}^{t} V(t-s)\left(T_{h} f(u)-\Pi T_{N h} f(u)\right) d s\right\|_{l^{5} L^{10}} \lesssim\left\|T_{h} f(u)-\Pi T_{N h} f(u)\right\|_{L^{5 / 4} l^{5 / 4}} \\
\lesssim h^{s}\left\||D|^{s} f(u)\right\|_{L_{t}^{5 / 4} L_{x}^{5 / 4}}
\end{aligned}
$$

and using again the chain rule with $p_{1}=10 / 3, p_{2}=2$ we find

$$
\begin{array}{r}
\left\|\int_{0}^{t} V(t-s)\left(T_{h} f(u)-\Pi T_{N h} f(u)\right) d s\right\|_{l^{5} L^{10}} \lesssim h^{s}\|\| u\left\|_{L_{x}^{5}}^{3 / 2}\right\||D|^{s} u\left\|_{L_{x}^{2}}\right\|_{L_{t}^{5 / 4}} \\
\lesssim\|u\|_{L_{x}^{5} L_{T}^{10}}^{3}+\|u\|_{L_{T}^{\infty} H_{x}^{s}}^{2} .
\end{array}
$$

For $I_{4}$, we have using (7.24)

$$
\begin{aligned}
\left\|\int_{0}^{t} V_{h} \Pi E\left(T_{h} f(u)-f\left(u_{h}\right)\right) d s\right\|_{l^{2}} & \lesssim\left\|T_{h} f(u)-f\left(T_{h} u\right)\right\|_{L^{1}\left([0, T] ; l^{2}\right)}+\left\|f\left(T_{h} u\right)-f\left(u_{h}\right)\right\|_{L^{1} l^{2}} \\
& \lesssim h^{s}\|u\|_{L_{T}^{1} W^{s, 5}}^{5 / 2}+\left\|f\left(T_{h} u\right)-f\left(u_{h}\right)\right\|_{L_{T}^{1} l^{2}} .
\end{aligned}
$$

Since $\left\|f\left(T_{h} u\right)-f\left(u_{h}\right)\right\|_{L_{T}^{1} l^{2}} \lesssim\left\|T_{h} u-u_{h}\right\|_{L^{2} l^{5}}\left(\left\|T_{h} u\right\|_{L^{3} l^{5}}^{3 / 2}+\left\|u_{h}\right\|_{L^{3} l^{5}}^{3 / 2}\right)$, using again Hölder's inequality in time, this term can be absorbed (for $T$ small enough independent of $h$ ) in the left 
hand side. The $l^{5} L^{10}$ norm of $I_{4}$ is dealt with in the same way:

$$
\begin{aligned}
\left\|\int_{0}^{t} V_{h} \Pi E\left(T_{h} f(u)-f\left(u_{h}\right)\right) d s\right\|_{l^{5} L^{10}} \lesssim & \| T_{h} f(u)-f\left(u_{h} \|_{L^{5 / 4} l^{5 / 4}}\right. \\
\lesssim & \left\|T_{h} f(u)-f\left(T_{h} u\right)\right\|_{L^{5 / 4} l^{5 / 4}}+t^{1 / 10}\left\|f\left(T_{h} u\right)-f\left(u_{h}\right)\right\|_{L^{5 / 4} l^{5 / 4}} \\
\lesssim & h^{s}\left(\left\||D|^{s} u\right\|_{l^{5} L^{10}}^{5 / 2}+\|u\|_{l^{5} L^{10}}+\|u\|_{L^{\infty} H^{s}}\right) \\
& +t^{1 / 10}\left\|T_{h} u-u_{h}\right\|_{L^{25 / 8}{ }^{25 / 8}}\left(\|u\|_{L^{25 / 8} l^{25 / 8}}+\left\|u_{h}\right\|_{L^{25 / 8} l^{25 / 8}}\right) \\
\lesssim & h^{s}\left(\left\|\left.D\right|^{s} u\right\|_{l^{5} L_{T}^{10}}^{5 / 2}+\|u\|_{l^{5} L_{T}^{10}}+\|u\|_{L_{T}^{\infty} H^{s}}\right) \\
& +T^{13 / 20}\left\|T_{h} u-u_{h}\right\|_{X_{T}}\left(\left\|u_{h}\right\|_{X_{h, T}}+\|u\|_{X_{T}}\right),
\end{aligned}
$$

(in this chain of inequality we implicitly used the continuity of $T_{h}: L^{p} \rightarrow l^{p}$ before interversion of time and space integration). For $T$ small enough the second term of the right hand side can be absorbed in the left hand side as in the proof of existence for the problem (7.20). Gluing all the estimates we have obtained

$$
\begin{array}{r}
\left\|T_{h} u-u_{h}\right\|_{l^{5} L_{T}^{10} \cap L_{T}^{\infty} l^{2}} \leq C h^{8 s / 25}\left(\|u\|_{L_{T}^{\infty} H^{s}}+\|u\|_{l^{5} L_{T}^{10}}+\left\||D|^{s} u\right\|_{l^{5} L_{T}^{10}}\right. \\
+\|u\|_{L_{T}^{\infty} H^{s}}^{5 / 2}+\|u\|_{l^{5} L_{T}^{10}}^{5 / 2}+\left\||D|^{s} u\right\|_{l^{5} L_{T}^{10}}^{5 / 2} .
\end{array}
$$

Remark 20. By continuity of $u$ with respect to the initial data, the estimate (7.22) could also be stated as

$$
\left\|u_{h}-T_{h} u\right\|_{X_{h, T}} \lesssim h^{8 s / 25}\left\|u_{0}\right\|_{H^{s}}
$$

\section{Some open questions and perspectives}

- The existence of rates of convergence for the approximation of the quasi-linear cKdV equation is still open. Basically one would need to obtain rates for every linear dispersive estimates, but the time-space integration may open some other difficulties,

- The schemes studied here are only semi-discrete, it is essential to introduce time discretization that do not break the dispersive estimates (this was done for NLS in [4]), and it would be of particular interest to compare them with more standard schemes when the initial data are sufficiently rough (only in $H^{s}$ for $s<3 / 2$ ),

- The construction of dispersive schemes that do not rely in some way on the Fourier transform seems so far way beyond reach. 


\section{A Standard results of harmonic analysis}

This section is devoted to some results of standard Fourier analysis that are maybe less known in discrete settings. The proofs are elementary adaptations from the ones in continuous settings and we include them only for completeness.

Definition 1. We define the (discrete) maximal function of a sequence $u$ as

$$
(\mathcal{M} u)_{n}=\sup _{k} \frac{1}{2 k+1} \sum_{j=n-k}^{n+k}\left|u_{j}\right| .
$$

The weak space $l_{w}^{1}(h \mathbb{Z})$ is the set of sequences such that $\exists C>0:\left|\left\{k:\left|u_{k}\right|>\alpha\right\}\right| \leq C / \alpha$. Here $|A|$ is $h$ times the cardinal of $A$.

Proposition 21. The maximal function satisfies the following properties:

- for $u \in l^{1}(h \mathbb{Z}), \alpha>0$,

$$
\left|\left\{k:(\mathcal{M} u)_{k}>\alpha\right\}\right| \lesssim\|u\|_{1} / \alpha .
$$

(i.e. $\mathcal{M}$ is continuous from $l^{1} \rightarrow l_{w}^{1}$ ).

- For $p>1, \mathcal{M}$ is continuous $l^{p} \rightarrow l^{p}$.

Proof. The second point is obvious for $p=\infty$. If the first point is proved, the general case for the second point is implied by the Marcinkiewicz interpolation theorem.

Thus we focus on the first point: fix $N \in \mathbb{N}^{*}, A_{\alpha, N}=\left\{|k| \leq N:(\mathcal{M} u)_{k}>\alpha\right\}$. For $k \in A_{\alpha, N}$, there exists $n_{k}$ such that

$$
\frac{1}{2 n_{k}+1} \sum_{k-n_{k}}^{k+n_{k}}\left|u_{k}\right|>\alpha
$$

The set $\cup_{k \in A_{\alpha, N}}\left[k-n_{k}, k+n_{k}\right]$ contains $A_{\alpha, N}$, from Vitali's covering lemma there exists a subset $\widetilde{A}$ of $A_{\alpha, N}$ such that $\cup_{k \in \widetilde{A}}\left[k-3 n_{k}, k+3 n_{k}\right] \supset A_{\alpha, N}$ and the $\left[k-n_{k}, k+n_{k}\right]$ are disjoint. Therefore:

$$
\left|A_{\alpha, N}\right| \leq 3 h \sum_{k \in \widetilde{A}} 2 n_{k}+1 \leq 3 h \sum_{k \in \widetilde{A}} \sum_{k-n_{k}}^{k+n_{k}} \frac{\left|u_{k}\right|}{\alpha} \leq 3 \frac{\|u\|_{l^{1}(h \mathbb{Z})}}{\alpha} .
$$

The estimate being uniform in $N$, this directly implies by letting $N \rightarrow \infty$

$$
\left|\left\{k:(\mathcal{M} u)_{k} \leq \alpha\right\}\right|=\left|A_{\alpha, \infty}\right| \lesssim \frac{\|u\|_{l^{1}(h \mathbb{Z})}}{\alpha},
$$

which is the continuity of $\mathcal{M}: l^{1} \mapsto l_{w}^{1}$. 
Proposition 22. (discrete Hardy-Littlewood-Sobolev with parameter) Let $u \in l^{p}(h \mathbb{Z}), 1<p<q<\infty, 0<\gamma<1$ where

$$
\frac{1}{q}=\frac{1}{p}-1+\gamma
$$

We set

$$
u *_{h} v:=h \sum_{\mathbb{Z}} u_{n-k} v_{k}
$$

If $|v| \leq \frac{C}{(h(1+|k|))^{\gamma}}$, then $\left(\widetilde{u_{n}}\right) \in l^{q}(h \mathbb{Z})$ with $\|\widetilde{u}\|_{l^{q}} \lesssim C\|u\|_{l^{p}}$.

Proof. We first reduce the proof to the case $h=1$ : we write $\|u\|_{l_{h}^{p}}^{p}=h \sum\left|u_{n}\right|^{p}$, while $\|u\|_{l^{p}}=$ $\sum\left|u_{n}\right|^{p}$. With those notations, and if the result is proved for $h=1$

$$
\begin{aligned}
\left\|u *_{h} v\right\|_{l^{q}}^{q}=h \sum_{n}\left|h \sum_{k} u_{n-k} v_{k}\right|^{q}=h^{q+1}\|u * v\|_{l^{q}}^{q} & \leq h^{q+1}\|u\|_{l^{p}}^{q} C^{q} / h^{\gamma q} \\
& =C^{q} h^{q+1}\|u\|_{l_{h}^{p}}^{q} h^{-(\gamma q+q / p)} \\
& =C^{q}\|u\|_{l_{h}^{p}}^{q} .
\end{aligned}
$$

Since $\left|\sum u_{n-k} v_{k}\right| \leq C \sum\left|u_{n-k}\right| /(1+|k|)^{\gamma}$, we may assume $u_{n} \geq 0, v_{n}=1 /(1+|n|)^{\gamma}$. Let us write for $N \in \mathbb{N}$ arbitrary

$$
\sum_{\mathbb{Z}} u_{n-k} \frac{1}{(1+|k|)^{\gamma}}=\sum_{|k| \leq N} u_{n-k} \frac{1}{(1+|k|)^{\gamma}}+\sum_{|k|>N} u_{n-k} \frac{1}{(1+|k|)^{\gamma}} .
$$

The second term is bounded thanks to the Hölder inequality

$$
\sum_{|k|>N} u_{n-k} \frac{1}{(1+|k|)^{\gamma}} \leq\|u\|_{l p}\left(\sum_{|k|>N} \frac{1}{(1+|k|)^{\gamma p^{\prime}}}\right)^{1 / p^{\prime}} \lesssim\|u\|_{l^{p}} N^{1 / p^{\prime}-\gamma}
$$

For the first term, set

$$
\left\{\begin{array}{l}
\alpha_{j}=\frac{1}{(1+j)^{\gamma}}-\frac{1}{(2+j)^{\gamma}}, 0 \leq j<N \\
\alpha_{N}=\frac{1}{(1+N)^{\gamma}}
\end{array}\right.
$$


One has

$$
\begin{aligned}
\sum_{|k| \leq N} u_{n-k} \frac{1}{(1+|k|)^{\gamma}} & =\sum_{|k| \leq N} \sum_{j=|k|}^{N} u_{n-k} \alpha_{j} \\
& =\sum_{j=0}^{N} \sum_{|k| \leq j} \alpha_{j} u_{n-k} \\
& \leq \sum_{j=0}^{N} \alpha_{j}(2 j+1)(\mathcal{M} u)_{n} \\
& =(\mathcal{M} u)_{n} \sum_{j=0}^{N} \sum_{|k| \leq n} \alpha_{j} \\
& =(\mathcal{M u} u)_{n} \sum_{|k| \leq N} \sum_{j=|k|}^{N} \alpha_{j}=(\mathcal{M} u)_{n} \frac{1}{(1+|k|)^{\gamma}} \lesssim(\mathcal{M} u)_{n} N^{1-\gamma}
\end{aligned}
$$

Thus

$$
\left|\widetilde{u}_{n}\right| \lesssim N^{1 / p^{\prime}-\gamma}\|u\|_{l^{p}}+N^{1-\gamma}(\mathcal{M} u)_{n}
$$

we optimize in $N$ by chosing $N^{1 / p^{\prime}-\gamma}\|u\|_{l^{p}} \sim N^{1-\gamma}(\mathcal{M} u)_{n}$, which gives

$$
N=\left\lfloor\left(\frac{\|u\|_{l^{p}}}{(\mathcal{M} u)_{n}}\right)^{p}\right\rfloor, \text { where }\lfloor\cdot\rfloor \text { denotes the integer part. }
$$

Note that $(\mathcal{M} u)_{n} \leq\|u\|_{l^{\infty}} \leq\|u\|_{l^{p}}$ is always true, thus $N \geq 1$ and

$$
\frac{1}{2}\left\lfloor\left(\frac{\|u\|_{l^{p}}}{(\mathcal{M} u)_{n}}\right)^{p}\right\rfloor \leq N \leq\left\lfloor\left(\frac{\|u\|_{l^{p}}}{(\mathcal{M} u)_{n}}\right)^{p}\right\rfloor .
$$

Injecting this in (A.4) we find

$$
\begin{aligned}
\left|\widetilde{u}_{n}\right| & \lesssim(\mathcal{M} u)_{n}\left(\|u\|_{l^{p}}^{p(1-\gamma)}(\mathcal{M} u)_{n}^{p(\gamma-1)}\right)+\|u\|_{l^{p}}(\mathcal{M} u)_{n}^{-p\left(1 / p^{\prime}-\gamma\right)}\|u\|_{l^{p}}^{p\left(1 / p^{\prime}-\gamma\right)} \\
& \lesssim\|u\|_{l^{p}}^{p(1-\gamma)}(\mathcal{M} u)_{n}^{p / q} .
\end{aligned}
$$

Proposition 21 finally gives:

$$
\left\|\widetilde{u}_{n}\right\|_{l^{q}} \lesssim\|u\|_{l^{p}}^{p(1-\gamma)}\|\mathcal{M} u\|_{l^{p}}^{p / q} \lesssim\|u\|_{l^{p}}
$$

Proposition 23. Let $\mathcal{F}$ be the usual Fourier transform on $L^{2}(\mathbb{R})$. For $p>1$, the operator $I:\left(u_{n}\right) \rightarrow \mathcal{F}^{-1}\left(\widehat{u} 1_{[-\pi / h, \pi / h]}\right)$ is continuous $l^{p}(h \mathbb{Z}) \rightarrow L^{p}(\mathbb{R})$. 
Proof. (Sketch of) Note that the case $p=2$ is simply the Parseval identity. By homogeneity we are reduced to the case $h=1$, and we prove that $I$ is continuous from $l^{1}$ to $L_{w}^{1}$, this implies the strong $L^{p}$ continuity thanks to the Marcinkiewicz interpolation theorem for $1<p<2$ and then by duality for $p>2$. The proof is derived from the continuity of the Hilbert transform, however the result goes back at least to Plancherel and Polya [15], 1937. A basic calculus shows that for $\left(u_{n}\right)$ rapidly decaying

$$
I u(x)=\frac{1}{\pi} \sum_{n} u_{n} \frac{\sin \pi(x-n)}{\pi(x-n)} .
$$

For $\lambda$ fixed we use the decomposition $\mathbb{Z}=\left(\sqcup I_{k}\right) \sqcup\left(\mathbb{Z} \backslash\left(\sqcup I_{k}\right)\right)$ such that

- for any $k, \lambda \leq 1 /\left|I_{k}\right| \sum_{I_{k}}\left|u_{n}\right| \leq 2 \lambda$, in particular $\left|\sqcup I_{k}\right| \leq\|u\|_{1} / \lambda$,

- for any $n \notin \sqcup I_{k},\left|u_{n}\right| \leq \lambda$.

(this is a discrete Calderon-Zygmund decomposition, see for example Stein [17] I.3).

We define $g_{n}=u_{n}$ if $n \notin \sqcup I_{k}, e^{i n \pi} /\left|I_{k}\right| \sum_{I_{K}} e^{i j \pi} u_{j}$ if $n \in I_{k}$. Clearly, $\left|g_{n}\right| \leq 2 \lambda,\left\|g_{n}\right\|_{l^{1}} \leq\left\|u_{n}\right\|_{l^{1}}$, and we may write

$$
u_{n}=b_{n}+g_{n},
$$

where $b_{n}=0$ if $n \notin \sqcup I_{k}$ and

$$
b_{n}=u_{n}-e^{i n \pi} /\left|I_{k}\right| \sum_{I_{k}} e^{i j \pi} u_{j} \text { for } n \in I_{k} .
$$

In particular $\|b\|_{l^{1}} \leq 2\|u\|_{l^{1}}$ and for any $k, \sum_{I_{k}} e^{ \pm i n \pi} b_{n}=0$. We remark then

$$
|\{x \in \mathbb{R}:|I u| \geq \lambda\}| \leq|\{x \in \mathbb{R}:|I g| \geq \lambda / 2\}|+|\{x \in \mathbb{R}:|I b| \geq \lambda / 2\}| .
$$

Using Chebychev's inequality for $p=2$ and the $L^{2}$ continuity, we obtain for the first set

$$
|\{x \in \mathbb{R}:|I g| \geq \lambda / 2\}| \leq 4\|I g\|_{L^{2}}^{2} / \lambda^{2} \leq 8 \lambda\|u\|_{1} / \lambda^{2}=8\|u\|_{1} / \lambda .
$$

We note $c_{k}$ the center of $I_{k}$ and we write abusively $2 I_{k}$ for the interval of center $c_{k}$ and twice larger than $I_{k}$. Chebychev's inequality implies for the second term

$$
\begin{aligned}
|\{x \in \mathbb{R}:|I b| \geq \lambda / 2\}| & \leq 2 \sum\left|I_{k}\right|+\left|\left\{x \in \mathbb{R} \backslash\left(\sqcup 2 I_{k}\right):|I b| \geq \lambda / 2\right\}\right| \\
& \leq 2 \sum\left|I_{k}\right|+\frac{2}{\lambda} \int_{\mathbb{R} \backslash\left(\sqcup I_{k}\right)}|I b| d x \\
& \leq \frac{2}{\lambda}\|u\|_{1}+\frac{2}{\lambda} \sum_{k} \int_{\mathbb{R} \backslash I_{k}}\left|\sum_{I_{k}} b_{n} \frac{\sin \pi(x-n)}{\pi(x-n)}\right| d x .
\end{aligned}
$$


Using now $\sum_{I_{k}} e^{ \pm i n \pi} b_{n}=0$ we obtain

$$
\begin{aligned}
\int_{\mathbb{R} \backslash I_{k}}\left|\sum_{I_{k}} b_{n} \frac{\sin \pi(x-n)}{\pi(x-n)}\right| d x & =\frac{1}{2} \int_{\mathbb{R} \backslash I_{k}}\left|\sum_{I_{k}} b_{n}\left(\frac{e^{i \pi(x-n)}}{\pi(x-n)}-\frac{e^{-i \pi(x-n)}}{\pi(x-n)}\right)\right| d x \\
& =\frac{1}{2} \int_{\mathbb{R} \backslash 2 I_{k}} \mid \sum_{I_{k}} b_{n}^{k}\left(\frac{e^{-i \pi n}}{\pi(x-n)}-\frac{e^{-i \pi n}}{\pi\left(x-c_{k}\right)}-\frac{e^{i \pi n}}{\pi(x-n)}\right. \\
& \left.\leq \int_{\mathbb{R} \backslash 2 I_{k}} \sum_{I_{k}}\left|b_{n}\right| \mid \frac{e^{i \pi n}}{\pi\left(x-c_{k}\right)}\right) \mid d x \\
& \leq 2 \sum_{I_{k}}\left|b_{n}\right| \int_{\mathbb{R} \backslash 2 I_{k}} \frac{\left|I_{k}\right|}{\pi(x-n)\left(x-c_{k}\right)^{2}} d x \\
& =\frac{4}{\pi} \sum_{I_{k}}\left|b_{n}\right| .
\end{aligned}
$$

So that by summing it all

$$
|\{x \in \mathbb{R}:|I u| \geq \lambda\}| \leq \frac{4+8 / \pi}{\lambda}\|u\|_{1} .
$$

\section{B Dispersive smoothing and rate of convergence}

We did not manage to obtain a version with rates of convergence for the estimate (17.8)

$$
\left\||D|^{2} \int_{0}^{t} V\left(t-t^{\prime}\right) \Pi g\left(t^{\prime}\right) d t^{\prime}\right\|_{l^{\infty} L^{2}} \lesssim\|\Pi g\|_{l^{1} L^{2}},
$$

which is one of the main points that prevented us from obtaining rates of convergence for the approximation of the $\mathrm{cKdV}$ equation. It seems like the deep technical problem is the fact that in the proof of (7.8), $t$ lies in an unbounded set and thus $V_{h}(t)-V(t)$ is certainly not small. More modestly this section describes how - by using basic Littlewood-Paley analysis - one may obtain a non optimal rate of convergence on

$$
\left.\| T_{h} \partial_{x} V(t) u_{0}-\partial_{h} V_{h}(t) \Pi T_{N h} u_{0}\right) \|_{l^{\infty} L^{2} .}
$$

To do so, we outline rapidly the main features of the Littlewood-Paley decomposition for sequences of $h \mathbb{Z}$, which are similar to the usual properties for functions. Let $\psi$ be a smooth 
compactly supported function such that $\operatorname{supp}(\psi) \subset[-2,2]$ and $\forall|x| \leq 1, \psi(x)=1$. We set $\varphi_{0}=\psi$ and for $j \geq 1$

$$
\varphi_{j}:=\psi\left(2^{-j} \cdot\right)-\psi\left(2^{-j+1} \cdot\right)
$$

For $u \in \mathcal{S}(h \mathbb{Z})$, the operators $\Delta_{j}$ are defined by

$$
\left.\widehat{\Delta_{j} u}\right|_{[-\pi / h, \pi / h]}=\varphi_{j} \widehat{u},
$$

as for interpolation operators we chose not to emphasize the dependance on $h$ (which appears notably in the fact that for $2^{j} \geq 4 \pi / h, \Delta_{j} u=0$ ).

Using the "quasi-orthogonality" $|j-k| \geq 2 \Rightarrow \Delta_{j} \Delta_{k}=0$, it is easily seen that $\|u\|_{l^{2}}^{2} \asymp$ $\sum\left\|\Delta_{j} u\right\|_{l^{2}}^{2}$, and more deeply a key feature of this decomposition are the so-called Bernstein inequalities.

Proposition 24. (Bernstein inequalities) The following estimates hold:

$$
\begin{array}{r}
\forall p \geq 2,\left\|\Delta_{j} u\right\|_{l^{p}} \leq C 2^{j(1 / 2-1 / p)}\left\|\Delta_{j} u\right\|_{l^{2}}, \\
\forall s \geq 0,\left\||D|^{s} \Delta_{j} u\right\|_{l^{2}} \leq C_{s} 2^{j s}\left\|\Delta_{j} u\right\|_{l^{2}},
\end{array}
$$

with $C, C_{s}$ independant of $h$, and the constant $C$ is also independent of $p \in[2, \infty]$.

The main result of this section is the following.

Proposition 25. Let $\Pi$ be an interpolator as in theorem [6. For $u_{0} \in L^{2}$, we have the homogeneous estimate

$$
\forall \varepsilon>0,\left\|\partial_{x} V(t) T_{h} u_{0}-\partial_{h} V_{h}(t) \Pi T_{N h} u_{0}\right\|_{l^{\infty} L^{2}([0, T])} \leq \frac{C h^{4 s / 13}(1+T)}{\sqrt{\varepsilon}}\left\|u_{0}\right\|_{H^{s+\varepsilon}(\mathbb{R})} .
$$

The constant $C$ only depends on $\Pi$.

Proof. We first split

$$
\begin{aligned}
\left\|\partial_{x} V(t) T_{h} u_{0}-\partial_{h} V_{h}(t) \Pi T_{N h} u_{0}\right\|_{l^{\infty} L^{2}([0, T]) \leq} & \left\|\left(V(t)-V_{h}(t)\right) \partial_{h} \Pi T_{N h} u_{0}\right\|_{l^{\infty} L^{2}([0, T])} \\
& +\left\|V(t)\left(\partial_{x} T_{h}-\partial_{h} \Pi T_{N h}\right) u_{0}\right\|_{l^{\infty} L^{2}([0, T])} \\
= & M_{1}+M_{2} .
\end{aligned}
$$

We will only focus on the derivation of an estimate for $M_{1}$, the other one being similar and simpler. For any $f$ defined on $h \mathbb{Z} \times[0, T]$,

$$
\begin{aligned}
\|f\|_{l^{\infty} L_{T}^{2}}^{2}=\sup _{n} \int_{0}^{T}\left(\sum_{j} \Delta_{j} f_{n}\right)^{2} d t & \lesssim \frac{1}{\varepsilon} \sup _{n} \int_{0}^{T} \sum_{j} 2^{2 \varepsilon j}\left|\Delta_{j} f_{n}\right|^{2} d t \\
& \leq \frac{1}{\varepsilon} \sum_{j} 2^{2 \varepsilon j} \sup _{n} \int_{0}^{T}\left|\Delta_{j} f_{n}\right|^{2} d t=\frac{1}{\varepsilon} \sum_{j} 2^{2 \varepsilon j}\left\|\Delta_{j} f_{n}\right\|_{l^{\infty} L_{T}^{2}}^{2} .
\end{aligned}
$$


Applying it to $M_{1}$ we have

$$
\left\|\left(V(t)-V_{h}(t)\right) \partial_{h} \Pi T_{N h} u_{0}\right\|_{l^{\infty} L^{2}([0, T])}^{2} \lesssim \frac{1}{\varepsilon} \sum_{j} 2^{2 \varepsilon j}\left\|\left(V(t)-V_{h}(t)\right) \partial_{h} \Delta_{j} \Pi T_{N h} u_{0}\right\|_{l^{\infty} L_{T}^{2}}^{2} .
$$

On one side the dispersive estimate (2.3) gives

$$
\left\|\left(V(t)-V_{h}(t)\right) \partial_{h} \Delta_{j} \Pi T_{N h} u_{0}\right\|_{l^{\infty} L_{T}^{2}} \lesssim\left\|\Delta_{j} \Pi T_{N h} u_{0}\right\|_{l^{2}},
$$

while Bernstein's inequality (B.2) combined with the inequality $\left|e^{i t \xi^{3}}-e^{i t p_{h}(\xi)}\right| \lesssim t|\xi|^{5} h^{2}$ implies

$$
\begin{aligned}
\left\|\left(V(t)-V_{h}(t)\right) \partial_{h} \Delta_{j} \Pi T_{N h} u_{0}\right\|_{l^{\infty} L_{T}^{2}} & \lesssim\left\|\left(V(t)-V_{h}(t)\right) \partial_{h} \Delta_{j} \Pi T_{N h} u_{0}\right\|_{L_{T}^{\infty} l^{\infty}} \\
& \leq 2^{j / 2} \sup _{[0, T]}\left\|\left(V(t)-V_{h}(t)\right) \partial_{h} \Delta_{j} \Pi T_{N h} u_{0}\right\|_{l^{2}} \\
& \leq 2^{j / 2+6 j} h^{2} T\left\|\Delta_{j} \Pi T_{N h} u_{0}\right\|_{l^{2}},
\end{aligned}
$$

so that by (7.3) with $\alpha=2 s / 13$ applied to (B.7), (B.6),

$$
\left\|\left(V(t)-V_{h}(t)\right) \partial_{h} \Delta_{j} \Pi T_{N h} u_{0}\right\|_{l^{\infty} L_{T}^{2}} \leq h^{4 s / 13} 2^{j s}\left\|\Delta_{j} \Pi T_{N h} u_{0}\right\|_{l^{2}} .
$$

By summing and using lemma 1 we find as expected

$$
M_{1}^{2} \leq \sum_{j} h^{8 s / 13} \frac{2^{2 j(s+\varepsilon)}}{\varepsilon}\left\|\Delta_{j} \Pi T_{N h} u_{0}\right\|_{l^{2}}^{2} \lesssim \frac{h^{8 s / 13}}{\varepsilon}\left\|u_{0}\right\|_{H^{s+\varepsilon}}^{2} .
$$

\section{Acknowledgements}

A large part of this work was pursued at the Basque Center for Applied Mathematics, in the frame of the FP7-246775 NUMERIWAVES project, Funded by the European Research Council Executive Agency (ERCEA) in the Advanced Grant 2009 subprogram.

The author thanks Enrique Zuazua for introducing him to the problem and stimulating discussions.

\section{References}

[1] F. M. Christ and M. I. Weinstein. Dispersion of small amplitude solutions of the generalized Korteweg-de Vries equation. J. Funct. Anal., 100(1):87-109, 1991.

[2] Arnaud Debussche and Jacques Printems. Numerical simulation of the stochastic Korteweg-de Vries equation. Phys. D, 134(2):200-226, 1999. 
[3] Arnaud Debussche and Jacques Printems. Convergence of a semi-discrete scheme for the stochastic Korteweg-de Vries equation. Discrete Contin. Dyn. Syst. Ser. B, 6(4):761-781 (electronic), 2006.

[4] Liviu I. Ignat. Fully discrete schemes for the Schrödinger equation. Dispersive properties. Math. Models Methods Appl. Sci., 17(4):567-591, 2007.

[5] Liviu I. Ignat and Enrique Zuazua. Convergence rates for dispersive approximation schemes to nonlinear schrodinger equations. J. Math. Pures Appl., to appear.

[6] Liviu I. Ignat and Enrique Zuazua. A two-grid approximation scheme for nonlinear Schrödinger equations: dispersive properties and convergence. C. R. Math. Acad. Sci. Paris, 341(6):381-386, 2005.

[7] Liviu I. Ignat and Enrique Zuazua. Numerical dispersive schemes for the nonlinear Schrödinger equation. SIAM J. Numer. Anal., 47(2):1366-1390, 2009.

[8] Tosio Kato. Perturbation theory for linear operators. Classics in Mathematics. SpringerVerlag, Berlin, 1995. Reprint of the 1980 edition.

[9] Carlos E. Kenig, Gustavo Ponce, and Luis Vega. Oscillatory integrals and regularity of dispersive equations. Indiana Univ. Math. J., 40(1):33-69, 1991.

[10] Carlos E. Kenig, Gustavo Ponce, and Luis Vega. Well-posedness of the initial value problem for the Korteweg-de Vries equation. J. Amer. Math. Soc., 4(2):323-347, 1991.

[11] Carlos E. Kenig, Gustavo Ponce, and Luis Vega. The Cauchy problem for the Korteweg-de Vries equation in Sobolev spaces of negative indices. Duke Math. J., 71(1):1-21, 1993.

[12] Carlos E. Kenig, Gustavo Ponce, and Luis Vega. Well-posedness and scattering results for the generalized Korteweg-de Vries equation via the contraction principle. Comm. Pure Appl. Math., 46(4):527-620, 1993.

[13] Felipe Linares and Gustavo Ponce. Introduction to nonlinear dispersive equations. Universitext. Springer, New York, 2009.

[14] M. Nixon. The discretized generalized Korteweg-de Vries equation with fourth order nonlinearity. J. Comput. Anal. Appl., 5(4):369-397, 2003.

[15] M. Plancherel and G. Pólya. Fonctions entières et intégrales de fourier multiples. Comment. Math. Helv., 10(1):110-163, 1937.

[16] Jacques Printems. The stochastic Korteweg-de Vries equation in $L^{2}(\mathbb{R})$. J. Differential Equations, 153(2):338-373, 1999. 
[17] Elias M. Stein. Harmonic analysis: real-variable methods, orthogonality, and oscillatory integrals, volume 43 of Princeton Mathematical Series. Princeton University Press, Princeton, NJ, 1993. With the assistance of Timothy S. Murphy, Monographs in Harmonic Analysis, III. 